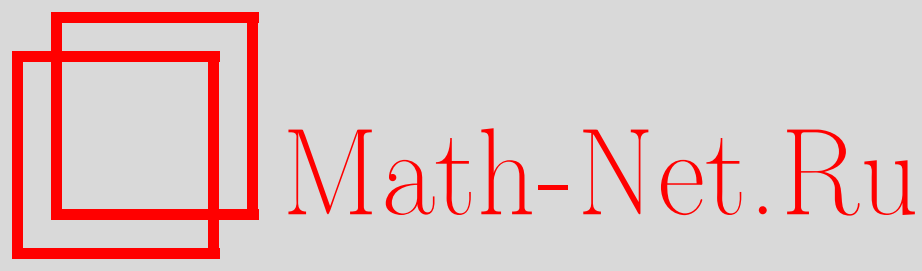

А. Л. Куземский, Метод неравновесного статистического оператора и обобщенные кинетические уравнения, TMФ, 2018, том 194, номер 1, 39-70

DOI: https://doi.org/10.4213/tmf9379

Использование Общероссийского математического портала Math-Net.Ru подразумевает, что вы прочитали и согласны с пользовательским соглашением http://www.mathnet.ru/rus/agreement

Параметры загрузки:

IP : 54.198 .67 .100

26 апреля 2023 г., 15:15:48

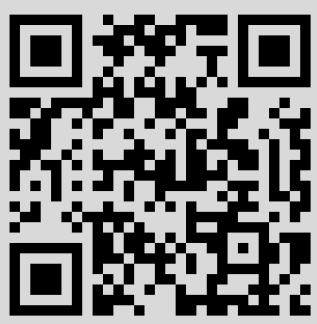




\title{
ФИЗИКА
}

Том 194, № 1

январь, 2018

(C) 2018 r.

А. Л. Куземский ${ }^{*}$

\section{МЕТОД НЕРАВНОВЕСНОГО СТАТИСТИЧЕСКОГО ОПЕРАТОРА И ОБОБЩЕННЫЕ КИНЕТИЧЕСКИЕ УРАВНЕНИЯ}

\begin{abstract}
В рамках подхода неравновесного статистического оператора Зубарева рассмотрены некоторые принципиальные вопросы неравновесной статистической термодинамики. Проведен краткий сравнительный анализ некоторых подходов к описанию необратимых процессов, основанному на концепции неравновесных ансамблей Гиббса, и их применимости для описания неравновесных процессов. Обсуждается вывод обобщенных кинетических уравнений для системы в термостате. Получено и проанализировано уравнение типа Шредингера с затуханием для динамической системы в термостате. Изучено динамическое поведение частицы в среде с учетом эффектов диссипации. Рассмотрена задача о рассеянии нейтронов в неравновесной среде и получена обобщенная формула Ван Хова. Показано, что метод неравновесного статистического оператора является эффективным и удобным инструментом для описания необратимых процессов в конденсированных средах.
\end{abstract}

Ключевые слова: неравновесная статистическая физика, необратимые процессы, метод неравновесного статистического оператора, открытые системы, обобщенные кинетические уравнения, уравнение типа Шредингера с затуханием, рассеяние нейтронов и обобщенная формула Ван Хова.

DOI: https://doi.org/10.4213/tmf9379

Работа посвящается памяти Д. Н. Зубарева, который был руководителем моей кандидатской диссертации и во многом определил круг моих интересов в дальнейшем

\section{1. ВВЕДЕНИЕ}

Целью статистической механики является создание последовательного и эффективного формализма для описания макроскопического поведения многочастичных систем на основе микроскопической теории. В статистической механике сформулирован весьма эффективный и надежный подход, позволяющий описывать большое

*Лаборатория теоретической физики им. Н.Н. Боголюбова, Объединенный институт ядерных исследований, Дубна, Московская обл., Россия. E-mail: kuzemsky@theor.jinr.ru 
многообразие явлений в таких системах, как жидкости, газы и твердые тела. Статистическая механика также дает обоснование таких термодинамических концепций, как теплота, температура и энтропия, на основе законов микроскопического поведения частиц.

Целью любой теории является описание реальности. Ясно, что реальные системы - это открытые системы, которые можно считать замкнутыми только приближенно. Центральной задачей неравновесной статистической механики является последовательный вывод уравнений эволюции данной системы исходя из обратимых уравнений движения. Именно неравновесная статистическая термодинамика дает подходы и инструменты для описания необратимых процессов в реальных системах в рамках единого теоретического метода, позволяя вычислять, хотя и приближенно, коэффициенты переноса, которые характеризуют развитие неравновесных процессов во времени.

Следует отметить, что один из основных законов термодинамики, а именно второе начало, отличается весьма существенным образом от других общих законов физики. Второе начало формулируется не в виде уравнения, но в форме неравенства. Это неравенство переходит в уравнение в предельном случае обратимых процессов. Однако известно, что на практике с фактической реализацией этого предела имеются трудности. По определению обратимый процесс - это процесс, при котором термодинамическая система никогда значительно не отклоняется от состояния равновесия. Поскольку любой процесс занимает конечное время, происходят неизбежные возмущения, влияющие на состояние равновесия. Поэтому весьма затруднительно вывести надежные и точные уравнения временной эволюции. Здесь стоит упомянуть о специфическом аспекте концепции времени в данном контексте: иногда говорят, что время в термодинамике проявляет себя не как величина, но только как некий индикатор (признак) особой величины, а именно изменения энтропии.

Для вычисления коэффициентов переноса существует много различных методов, которые, как правило, ограничены определенными условиями применимости. Например, наиболее широко используемый подход, основанный на уравнении Больцмана, применим для сильно разреженного газа точечных частиц.

В последние десятилетия были предложены различные схемы описания кинетических явлений и процессов переноса [1]-[13]. Это направление исследований активно развивается и имеет много различных аспектов [14]--[18]. Большой вклад в разработку современных методов равновесной и неравновесной статистической механики был внесен работами академика Боголюбова [1]-[3]. Далее мы обсудим кратко некоторые из этих подходов, в основном те, в которых используется метод неравновесных ансамблей Гиббса. При этом мы сосредоточим основное внимание на методе неравновесного статистического оператора (HCO), разработанном Зубаревым [5], [14], [15], и получении в рамках этого подхода обобщенных кинетических уравнений.

\section{2. МЕТОД РАВНОВЕСНЫХ И НЕРАВНОВЕСНЫХ АНСАМБЛЕЙ}

Задачей статистической механики является теоретическое описание наблюдаемых равновесных и неравновесных свойств систем многих частиц на основе их молекулярного состава, специфики межмолекулярного взаимодействия и природе их внешнего окружения [19], [20]. Универсальный аппарат равновесной статистической механики был развит Гиббсом [21] и называется методом ансамблей Гиббса. Метод 
Гиббса обладает большой степенью общности и весьма широкой областью применимости в задачах равновесной статистической механики. Концепции и подходы этого метода находят разнообразные применения в различных областях [15], [16].

Ансамбль характеризуется функцией распределения $f(\mathbf{p}, \mathbf{q})$, которая в случае классической механики должна удовлетворять уравнению Лиувилля

$$
\frac{d f}{d t}=0
$$

Это динамическое требование отражает тот факт, что точки в фазовом пространстве $(\mathbf{p}, \mathbf{q})$, представляющие состояния системы в ансамбле, не взаимодействуют между собой; при этом объем фазового пространства сохраняется. Метод ансамблей также включает в себя предположение о равных вероятностях микросостояний изолированных систем [15], [22], [23].

Нужно отметить, что понятия распределения Гиббса [24] и гиббсовского состояния играют важную роль при рассмотрении равновесных свойств статистических ансамблей. Распределение Гиббса в теории вероятностей и математической статистической механике [15], [16], [22], [23] определяется как распределение вероятностей, описывающее статистику конфигураций многочастичной системы в термодинамическом равновесии. Оно является инвариантным при дальнейшей эволюции системы. Таким образом, для систем в состоянии статистического равновесия имеется функция распределения Гиббса, с помощью которой возможно вычислить среднее значение по ансамблю для любой динамической величины. В отличие от этого случая, для необратимых процессов не было сформулировано подобного универсального распределения. Применялись многочисленные различные приближенные модели и методы [5], [15], [16], при этом рассматривались механические и тепловые возмущения системы, вызывающие неравновесные процессы [20], [15], [18]. Механические возмущения можно выразить как определенные вклады в гамильтониан системы; при этом отклонение от равновесия, вызванное подобными вкладами, может быть описано на основе теории возмущений.

Метод линейного отклика, развитый Кубо [5], [15], [16], [20], позволяет вычислять коэффициенты переноса при механических возмущениях, таких как внешнее электрическое поле. Это дает возможность найти компактное выражение для электропроводности в терминах корреляционных функций. В то же время такие тепловые возмущения, как градиенты плотности и температуры, нельзя непротиворечивым образом выразить в виде возмущающих вкладов в гамильтониане.

В стандартном термодинамическом подходе для описания свойств однородной равновесной системы используется небольшое число параметров, макроскопически определяющих ансамбль [5]. Для описания необратимых процессов в системах, которые не очень значительно отклоняются от состояния термодинамического равновесия, можно использовать следующий прием. Предполагается, что можно разбить систему на определенные малые подсистемы, каждая из которых находится в локально равновесном состоянии [5], [15], [16]. Другими словами, каждую такую подсистему можно рассматривать как отдельную термодинамическую систему, характеризующуюся небольшим числом физических переменных, описывающих ее состояние.

В статистической механике процессов переноса обычно рассматриваются две связанные между собой задачи, а именно выяснение того, каков механизм приближения 
к равновесию, и представление микроскопических свойств в терминах макроскопических потоков. Одним из эффективных подходов к данным проблемам является подход Цванцига [8], [9], [10], [25]. Он базируется на переформулировке метода Гиббса и использовании проекционных операторов в пространстве всех возможных плотностей ансамбля (или функций распределения), для того чтобы разделить плотность ансамбля на релевантную часть, позволяющую вычислять средние по ансамблю для определенных величин, и остающуюся, нерелевантную часть. Данное разбиение является обобщением известного разделения матрицы плотности на диагональную и недиагональную части, которое обычно используется при выводе управляющего кинетического уравнения (master equation). Было показано, что релевантная часть удовлетворяет кинетическому уравнению, являющемуся обобщением ранее полученных управляющих уравнений, в частности уравнения Ван Хова.

Подход, связанный с построением неравновесных ансамблей, развивался Бергманом и Лебовичем [26]-[29]. Существует много способов построения таких ансамблей, поэтому функция распределения $\rho\left(\mathbf{r}_{1}, \ldots, \mathbf{r}_{n}, \mathbf{p}_{1}, \ldots, \mathbf{p}_{n}, t\right)$, характеризующая состояние ансамбля, не определена однозначно. Данная функция должна быть выбрана так, чтобы среднее значение по ансамблю находилось в точном соответствии с неполной информацией о состоянии системы в заданное время. Дальнейшая эволюция системы во времени моделируется как поведение, усредненное по всем членам репрезентативного ансамбля.

Точное определение неравновесного состояния системы представляет собой весьма сложную задачу, поскольку это состояние не характеризуется однозначным образом. Однако в ряде случаев тщательное рассмотрение приводит к установлению различных временных шкал в процессе эволюции системы [1]-[3], [5]. Кроме того, часто можно считать локально равновесное (квазиравновесное) распределение и неравновесное распределение схожими по структуре и функциональной зависимости от параметров системы. Данное обстоятельство ведет к тому, что появляется возможность так называемого сокращенного описания, в рамках которого весьма обширный класс процессов переноса можно описать приближенно, используя определенное конечное число макроскопических величин, или релевантных переменных [5]. При этом необходимо иметь в виду, что для того чтобы система могла достичь устойчивого состояния или находиться в неравновесном стационарном состоянии, она не должна быть изолированной, но должна быть в контакте с окружающей средой (системой тепловых резервуаров), что обеспечивает наличие температурных и иных градиентов в системе. Это подчеркивалось в работах Бергмана и Лебовича [26]-[29], где были найдены неравновесные решения для некоторых простых систем в рамках подхода неравновесных ансамблей и предложен простой метод (релаксационного типа) для нахождения приближенных стационарных решений. Однако данная программа оказалась не вполне успешной. Основное неудобство этого подхода заключалось в необходимости детального оперирования взаимодействием между системой и резервуаром.

Другой метод, более близкий к методу НСО, развивался в работах Мак Леннана [6]. Он основан на введении внешних (неконсервативных) сил, которые описывают влияние окружения или теплового резервуара (термостата) на данную систему. Другими словами, рассматриваются конечные реальные возмущения, вызываемые термостатом, что и приводит к необратимому поведению системы. В большом чис- 
ле работ по данной тематике подчеркивалась необходимость разработки последовательной теории, описывающей сглаживание (smoothing) функции распределения в фазовом пространстве, которое дает возможность учитывать возрастание энтропии в открытых неравновесных системах. Другими словами, важную роль играет соответствующее усреднение функции распределения (статистического оператора). Эти вопросы имеют большое значение при изучении эргодичности динамических и статистических систем [5], [15], [16]. В этом контексте следует отметить, что эффективные методы усреднения при описании сложных (нелинейных) систем разрабатывались Боголюбовым и другими авторами [30]-[33]. В работе [34] отмечалось, что свойство перемешивания, возникающее в эргодической теории, не является необходимым условием для статистических систем при любых конечных значениях объема и числа частиц. Важным является только соответствующее поведение предельных средних значений макроскопических величин при $t \rightarrow \infty$ после термодинамического предельного перехода [5], [15], [16]. В работах [30], [31] в рамках теории возмущений по малому параметру $\varepsilon$ был разработан эффективный метод усреднения, позволяющий получать асимптотические решения дифференциального уравнения без секулярных членов. В этом подходе основной целью являлся поиск преобразования переменных, позволяющего разделить их на медленные и быстрые.

Весьма эффективным примером даного подхода явилась работа Боголюбова и Зубарева [35]. Метод усреднения, предложенный в работах [30], [31], получил широкое распространение при использовании в различных задачах теории динамических систем [30]-[33], а также в задачах статистической механики и теории конденсированных сред [5], [15], [16]. Эти идеи оказали также стимулирующее влияние на разработку метода НСО [5]. Данный метод органически включает в себя идеи о наличии в многочастичных системах разных временных и энергетических шкал и соответствующем сокращенном описании с помощью небольшого набора релевантных переменных [5]. При этом большую роль играет процедура усреднения по времени, описывающая сглаживание функции распределения [5] и набора релевантных переменных.

\section{3. МЕТОД НСО}

Основная идея метода НСО [5], [14] состоит в том, что при не слишком малых масштабах времени для описания неравновесного состояния системы достаточно некоторого набора параметров $\left\{F_{m}(t)\right\}$, и при этом можно найти частное решение $\rho(t, 0)$ уравнения Лиувилля (2.1), зависящее от времени лишь через величины $F_{m}(t)$. Первый аргумент функции $\rho(t, 0)$ указывает на неявную зависимость от времени. Предполагается, что неравновесный статистический ансамбль можно охарактеризовать небольшим набором релевантных операторов $P_{m}(t)$ (квазиинтегралов движения); при этом НСО является функционалом от них,

$$
\rho(t)=\rho\left\{\ldots, P_{m}(t), \ldots\right\}
$$

Можно показать [5], что если статистический оператор $\rho(t, 0)$ удовлетворяет уравнению Лиувилля, то он совпадает с

$$
\rho(t)=\exp \left(\Lambda(t)-\int_{-\infty}^{0} d t_{1} \sum_{m} G_{m}\left(t, t_{1}\right) P_{m}\left(t_{1}\right)\right), \quad \Lambda(t)=1-\lambda(t),
$$


где

$$
\begin{gathered}
G_{m}\left(t, t_{1}\right)=\varepsilon e^{\varepsilon t_{1}} F_{m}\left(t+t_{1}\right), \\
\Lambda(t)=\varepsilon \int_{-\infty}^{0} d t_{1} e^{\varepsilon t_{1}} \lambda\left(t+t_{1}\right)=\lambda(t)-\int_{-\infty}^{0} d t_{1} e^{\varepsilon t_{1}} \dot{\lambda}\left(t+t_{1}\right) .
\end{gathered}
$$

Перепишем формулу (3.2) в другом виде:

$$
\begin{aligned}
\rho & =\exp \left(\overline{\ln \rho_{\mathrm{q}}(t)}\right)=\exp \left(\varepsilon \int_{-\infty}^{0} d t_{1} e^{\varepsilon t_{1}} \exp \left(\frac{i H t_{1}}{\hbar}\right) \ln \rho_{\mathrm{q}}\left(t+t_{1}\right) \exp \left(\frac{-i H t_{1}}{\hbar}\right)\right)= \\
& =\exp (-\overline{S(t, 0)})=\exp \left(-\varepsilon \int_{-\infty}^{0} d t_{1} e^{\varepsilon t_{1}} S\left(t+t_{1}, t_{1}\right)\right)= \\
& =\exp \left(-S(t, 0)+\int_{-\infty}^{0} d t_{1} e^{\varepsilon t_{1}} \dot{S}\left(t+t_{1}, t_{1}\right)\right)
\end{aligned}
$$

где черта сверху обозначает взятие инвариантной части оператора [5] и

$$
\begin{gathered}
\rho_{\mathrm{q}}(t)=\exp \left(\Omega(t)-\sum_{m} F_{m}(t) P_{m}\right) \equiv \exp (-S(t, 0)), \\
\Omega(t)=\ln \operatorname{Tr} \exp \left(-\sum_{m} F_{m}(t) P_{m}\right)
\end{gathered}
$$

a $S\left(t, t_{1}\right)$ удовлетворяет уравнениям

$$
\begin{aligned}
\dot{S}(t, 0) & =\frac{\partial S(t, 0)}{\partial t}+\frac{1}{i \hbar}[S(t, 0), H] \\
\dot{S}\left(t, t_{1}\right) & =\exp \left(\frac{i H t_{1}}{\hbar}\right) \dot{S}(t, 0) \exp \left(\frac{-i H t_{1}}{\hbar}\right)
\end{aligned}
$$

Здесь $\rho_{\mathrm{q}}-$ квазиравновесный статистический оператор, соответствующий экстремальному значению информационной энтропии $S=-\operatorname{Tr}(\rho \ln \rho)$ [5], [15], [36]-[39] при дополнительных условиях постоянства $\operatorname{Tr}\left(\rho P_{m}\right)=\left\langle P_{m}\right\rangle_{\mathrm{q}}, \operatorname{Tr} \rho=1$. В этом случае

$$
\frac{\delta \Phi}{\delta F_{m}}=-\left\langle P_{m}\right\rangle_{\mathrm{q}}, \quad\langle\cdot\rangle_{\mathrm{q}}=\operatorname{Tr}\left(\rho_{\mathrm{q}} \bullet\right),
$$

где

$$
\Phi(\rho)=-\operatorname{Tr}(\rho \ln \rho)-\sum_{m} F_{m} \operatorname{Tr}\left(\rho P_{m}\right)+\lambda \operatorname{Tr} \rho, \quad\left\langle P_{m}\right\rangle^{t}=\left\langle P_{m}\right\rangle_{\mathrm{q}}^{t}
$$

Подчеркнем еще раз, что, как уже говорилось, метод НСО существенным образом опирается на аналогию с нелинейной механикой [30]-[33]. Нелинейная система, стремящаяся к предельному циклу, “забывает" о своих начальных условиях. Поэтому необходимые переменные, характеризующие систему (релевантные операторы), которые зависят от времени посредством $F_{m}(t)$, должны конструироваться путем взятия части от операторов, входящих в логарифм статистического оператора, инвариантной по отношению к движению с гамильтонианом $H$. 
Поясним сказанное подробнее. По определению взятие инвариантной части оператора $F_{m}(t) P_{m}$ дает следующий результат:

$$
\begin{aligned}
B_{m}(t) & =\overline{F_{m}(t) P_{m}}=\varepsilon \int_{-\infty}^{0} d t_{1} e^{\varepsilon t_{1}} F_{m}\left(t+t_{1}\right) P_{m}\left(t_{1}\right)= \\
& =F_{m}(t) P_{m}-\int_{-\infty}^{0} d t_{1} e^{\varepsilon t_{1}}\left(F_{m}\left(t+t_{1}\right) \dot{P}_{m}\left(t_{1}\right)+\dot{F}_{m}\left(t+t_{1}\right) P_{m}\left(t_{1}\right)\right) .
\end{aligned}
$$

Здесь $\varepsilon \rightarrow 0$ и

$$
\dot{P}_{m}=\frac{1}{i \hbar}\left[P_{m}, H\right], \quad \dot{F}_{m}(t)=\frac{d F_{m}(t)}{d t} .
$$

Нужно подчеркнуть, что параметр $\varepsilon>0$ мы устремляем к нулю только после термодинамического предельного перехода [22], [23]. Операторы $B_{m}(t)$ удовлетворяют уравнению Лиувилля в пределе $\varepsilon \rightarrow 0$ :

$$
\frac{\partial B_{m}}{\partial t}-\frac{1}{i \hbar}\left[B_{m}, H\right]=\varepsilon \int_{-\infty}^{0} d t_{1} e^{\varepsilon t_{1}}\left(F_{m}\left(t+t_{1}\right) \dot{P}_{m}\left(t_{1}\right)+\dot{F}_{m}\left(t+t_{1}\right) P_{m}\left(t_{1}\right)\right) .
$$

Таким образом, операция взятия инвариантной части приводит к сглаживанию осциллирующих членов.

Заметим, что схожая процедура используется в формальной теории рассеяния [5] и позволяет наложить такие граничные условия, которые приводят к исключению опережающих решений уравнения Шредингера [5], [15]. Это особенно ясно видно, когда параметры $F_{m}(t)$ не зависят от времени. Дифференцирование оператора $\overline{P_{m}}$ по времени дает

$$
\frac{\partial \overline{P_{m}(t)}}{\partial t}=\varepsilon \int_{-\infty}^{0} d t_{1} e^{\varepsilon t_{1}} \dot{P}_{m}\left(t+t_{1}\right) .
$$

Величины $\overline{P_{m}(t)}$ можно считать интегралами (или квазиинтегралами) движения, хотя они сохраняются только в пределе $\varepsilon \rightarrow 0$. В данном контексте эта процедура ведет к отбору только запаздывающих решений уравнения Лиувилля.

Квантовое уравнение Лиувилля (как и классическое) инвариантно по отношению к изменению знака времени. Известно, что в равновесной статистической механике очень большое значение имеет концепция Боголюбова о квазисредних [40]-[42]. Согласно этой концепции бесконечно малые возмущения могут оказывать существенные воздействия на систему, если они нарушают какую-либо симметрию, снимая вырождение (или квазивырождение). В результате их влияние может быть конечным при условии их стремления к нулю после термодинамического предельного перехода. Зубаревым было показано [41], что концепция нарушающих симметрию возмущений и квазисредних также имеет существенное значение для теории необратимых процессов [5]. Метод построения НСО [5] в рамках представления о квазисредних приобретает большую прозрачность и глубину. Основная идея работы [41] состояла в том, что рассматривались бесконечно малые источники, нарушающие симметрию уравнения Лиувилля.

Можно показать [41], что $\rho(t, 0)$ удовлетворяет уравнению Лиувилля, но с бесконечно малым источником в правой части, пропорциональным величине $\varepsilon$, которая 
устремляется к нулю после термодинамического предельного перехода. В самом деле, рассмотрим уравнение

$$
\frac{\partial \rho_{\varepsilon}}{\partial t}+\frac{1}{i \hbar}\left[\rho_{\varepsilon}, H\right]=-\varepsilon\left(\rho_{\varepsilon}-\rho_{\mathrm{q}}\right)
$$

или, в другой форме,

$$
\frac{\partial \ln \rho_{\varepsilon}}{\partial t}+\frac{1}{i \hbar}\left[\ln \rho_{\varepsilon}, H\right]=-\varepsilon\left(\ln \rho_{\varepsilon}-\ln \rho_{\mathrm{q}}\right),
$$

где $\varepsilon \rightarrow 0$ после термодинамического предельного перехода. Уравнение (3.13) является аналогом соответствующего уравнения в квантовой теории рассеяния [5]. Введение бесконечно малых источников в уравнение Лиувилля соответствует наложению граничных условий

$$
\exp \left(\frac{i H t_{1}}{\hbar}\right)\left(\rho\left(t+t_{1}\right)-\rho_{\mathrm{q}}\left(t+t_{1}\right)\right) \exp \left(-\frac{i H t_{1}}{\hbar}\right) \rightarrow 0 .
$$

Здесь $t_{1} \rightarrow-\infty$ после термодинамического предельного перехода.

В работах [43]-[45] было показано, что оператор $\rho_{\varepsilon}$ имеет вид

$$
\rho_{\varepsilon}(t, t)=\varepsilon \int_{-\infty}^{t} d t_{1} e^{\varepsilon\left(t_{1}-t\right)} \rho_{\mathrm{q}}\left(t_{1}, t_{1}\right)=\varepsilon \int_{-\infty}^{0} d t_{1} e^{\varepsilon t_{1}} \rho_{\mathrm{q}}\left(t+t_{1}, t+t_{1}\right) .
$$

Здесь первый аргумент функции обозначает косвенную зависимость от времени через параметры $F_{m}(t)$, а второй - зависимость от времени через представление Гейзенберга. Искомый статистический оператор определяется следующим образом:

$$
\rho_{\varepsilon}=\rho_{\varepsilon}(t, 0)=\overline{\rho_{\mathrm{q}}(t, 0)}=\varepsilon \int_{-\infty}^{0} d t_{1} e^{\varepsilon t_{1}} \rho_{\mathrm{q}}\left(t+t_{1}, t_{1}\right) .
$$

В результате НСО принимает вид

$$
\begin{aligned}
\rho & =Q^{-1} \exp \left(-\sum_{m} B_{m}\right)=Q^{-1} \exp \left(-\sum_{m} \varepsilon \int_{-\infty}^{0} d t_{1} e^{\varepsilon t_{1}} F_{m}\left(t+t_{1}\right) P_{m}\left(t_{1}\right)\right)= \\
& =Q^{-1} \exp \left(-\sum_{m} F_{m}(t) P_{m}+\sum_{m} \int_{-\infty}^{0} d t_{1} e^{\varepsilon t_{1}}\left(\dot{F}_{m}\left(t+t_{1}\right) P_{m}\left(t_{1}\right)+F_{m}\left(t+t_{1}\right) \dot{P}_{m}\left(t_{1}\right)\right)\right) .
\end{aligned}
$$

Запишем уравнение (3.14) в следующей форме:

$$
\frac{d}{d t}\left(e^{\varepsilon t} \ln \rho(t, t)\right)=\varepsilon e^{\varepsilon t} \ln \rho_{\mathrm{q}}(t, t),
$$

где

$$
\ln \rho(t, t)=U^{\dagger}(t, 0) \ln \rho(t, 0) U(t, 0), \quad U(t, 0)=\exp \left(\frac{i H t}{\hbar}\right) .
$$

После интегрирования уравнения (3.19) в интервале $(-\infty, 0)$ получим

$$
\ln \rho(t, t)=\varepsilon \int_{-\infty}^{0} d t_{1} e^{\varepsilon t_{1}} \ln \rho_{\mathrm{q}}\left(t+t_{1}, t+t_{1}\right)
$$


Предполагается, что $\lim _{\varepsilon \rightarrow+0} \ln \rho(t, t)=0$. В результате имеем

$$
\rho(t, 0)=\exp \left(-\varepsilon \int_{-\infty}^{0} d t_{1} e^{\varepsilon t_{1}} \ln \rho_{\mathrm{q}}\left(t+t_{1}, t_{1}\right)\right)=\exp \left(\overline{\ln \rho_{\mathrm{q}}(t, 0)}\right) \equiv \exp (\overline{-S(t, 0)}) .
$$

Среднее значение любой динамической переменной $A$ вычисляется как

$$
\langle A\rangle=\lim _{\varepsilon \rightarrow+0} \operatorname{Tr}(\rho(t, 0) A)
$$

и видно, что оно фактически является не чем иным, как квазисредним. Нормировка квазиравновесного распределения $\rho_{\mathrm{q}}$ будет сохраняться при условиях

$$
\operatorname{Tr}\left(\rho(t, 0) P_{m}\right)=\left\langle P_{m}\right\rangle=\left\langle P_{m}\right\rangle_{\mathrm{q}}, \quad \operatorname{Tr} \rho=1 .
$$

Таким образом, можно утверждать, что появление эффекта необратимости тесно связано с нарушением симметрии времени [15], а также, в определенном смысле, с идеей квазисредних в статистической механике [40]-[42].

Прежде чем перейти к обсуждению кинетических уравнений, обсудим кратко иерархию временных шкал в неравновесных процессах. Одним из существенных преимуществ метода НСО является то, что в нем существование различных временных шкал подчеркивается с самого начала. Предположим, что гамильтониан нашей системы можно представить в виде $H=H_{0}+V$, где $H_{0}$ является невозмущенным гамильтонианом и $V$ описывает слабое возмущение. Такое разделение исходного гамильтониана не является вполне однозначным и зависит от специфики изучаемой системы. Существенно, что выбор оператора $H_{0}$ определяет короткую временную шкалу $\tau_{0}$. Этот выбор определяется тем обстоятельством, что для времен $t \gg \tau_{0}$ неравновесное состояние системы можно с разумной степенью точности описать с помощью средних значений некоторого конечного набора операторов $P_{m}$.

Предполагается, что после короткого времени $\tau_{0}$ (быстрая стадия) система может достигать состояния неполного или квазиравновесного состояния. При этом основное предположение состоит в том, что состояние системы полностью определяется с помощью квазиинтегралов движения, которые являются внутренними параметрами системы. Характерные релаксационные времена этих параметров много больше чем $\tau_{0}$. Тогда с большой степенью правдоподобия можно считать, что даже если эти квазиинтегралы в начальный момент времени не имели определенных равновесных значений, то по прошествии времени $\tau_{0}$ в квазиравновесном состоянии эти быстро меняющиеся переменные становятся функциями внешних параметров и квазиинтегралов движения. Важно подчеркнуть, что данная функциональная связь не зависит от начальных значений этих переменных. Другими словами, операторы $P_{m}$ выбираются так, чтобы они удовлетворяли условию

$$
\left[P_{k}, H_{0}\right]=\sum_{l} c_{k l} P_{l},
$$

где $c_{k l}$-некоторые коэффициенты (комплексные числа). Только для этого набора релевантных операторов следует записывать уравнения переноса. Отметим, что релевантные операторы могут быть скалярами и векторами.

Уравнения движения средних значений других (нерелевантных) операторов (других физических переменных) будут в некотором смысле следствием уравнений переноса для релевантных операторов; при этом соотношение (3.24) ведет к бесконечной 
цепочке операторных равенств. Для времен $t \leqslant \tau_{0}$ неравновесные средние от этих операторов быстро осциллируют, а для времен $t>\tau_{0}$ они становятся функциями их средних значений.

\section{4. ОБОБЩЕННЫЕ КИНЕТИЧЕСКИЕ УРАВНЕНИЯ}

Метод НСО [5] получил широкое применение в различных задачах статистической механики. Большой вклад в разработку теории кинетических уравнений в рамках метода НСО был внесен Покровским [46]. Обобщенные кинетические уравнения переноса, описывающие эволюцию переменных $\left\langle P_{m}\right\rangle$ и $F_{m}(t)$ во времени, получаются путем усреднения уравнений движения для $P_{m}$ с найденным $\mathrm{HCO}$

$$
\left\langle P_{m}\right\rangle=-\frac{\delta \Omega}{\delta F_{m}(t)}, \quad F_{m}(t)=\frac{\delta S}{\delta\left\langle P_{m}\right\rangle} .
$$

Обобщенные уравнения переноса имеют вид

$$
\left\langle\dot{P}_{m}\right\rangle=-\sum_{n} \frac{\delta^{2} \Omega}{\delta F_{m}(t) \delta F_{n}(t)} \dot{F}_{n}(t), \quad \dot{F}_{m}(t)=\sum_{n} \frac{\delta^{2} S}{\delta\left\langle P_{m}\right\rangle \delta\left\langle P_{n}\right\rangle}\left\langle\dot{P}_{n}\right\rangle .
$$

При этом производство энтропии записывается следующим образом:

$$
\dot{S}(t)=\langle\dot{S}(t, 0)\rangle=-\sum_{m}\left\langle\dot{P}_{m}\right\rangle F_{m}(t)=-\sum_{n, m} \frac{\delta^{2} \Omega}{\delta F_{m}(t) \delta F_{n}(t)} \dot{F}_{n}(t) F_{m}(t) .
$$

Уравнения (4.2) являются взаимно сопряженными и вместе с уравнением (4.3) образуют полную систему уравнений для вычисления величин $\left\langle P_{m}\right\rangle$ и $F_{m}$.

Следуя работе [46], запишем кинетические уравнения для системы со слабым взаимодействием. Гамильтониан имеет вид $H=H_{0}+V$, где $H_{0}$ - гамильтониан невзаимодействующих частиц (или квазичастиц), $V$ - оператор взаимодействия. В качестве релевантных операторов выберем набор операторов $P_{k}$ в виде $a_{k}^{\dagger} a_{k}$ или $a_{k}^{\dagger} a_{k+q}$. Здесь $a_{k}^{\dagger}$ и $a_{k}$ - операторы рождения или уничтожения (Ферми или Бозе). Исходными являются уравнения движения

$$
\dot{P}_{k}=\frac{1}{i \hbar}\left[P_{k}, H\right]
$$

В дальнейшем будем считать что выполняется соотношение (3.24).

В соответствии с (3.18) имеем

$$
\rho=Q^{-1} \exp \left(-\sum_{k} F_{k}(t) P_{k}+\sum_{k} \int_{-\infty}^{0} d t_{1} e^{\varepsilon t_{1}}\left(\dot{F}_{k}\left(t+t_{1}\right) P_{k}\left(t_{1}\right)+F_{k}\left(t+t_{1}\right) \dot{P}_{k}\left(t_{1}\right)\right)\right) .
$$

При этом мы учитываем, что $\left\langle P_{k}\right\rangle=\left\langle P_{k}\right\rangle_{\mathrm{q}}$. Обобщенные кинетические уравнения [46] для $\left\langle P_{k}\right\rangle$ записываются в виде

$$
\frac{d\left\langle P_{k}\right\rangle}{d t}=\frac{1}{i \hbar}\left\langle\left[P_{k}, H\right]\right\rangle=\frac{1}{i \hbar} \sum_{l} c_{k l}\left\langle P_{l}\right\rangle+\frac{1}{i \hbar}\left\langle\left[P_{k}, V\right]\right\rangle .
$$


Правые части этих уравнений содержат обобщенный интеграл столкновений, который можно записать с помощью разложения по степеням $V$ в виде

$$
\frac{d\left\langle P_{k}\right\rangle}{d t}=L_{k}^{0}+L_{k}^{1}+L_{k}^{21}+L_{k}^{22}
$$

где

$$
\begin{gathered}
L_{k}^{0}=\frac{1}{i \hbar} \sum_{l} c_{k l}\left\langle P_{l}\right\rangle_{\mathrm{q}}, \quad L_{k}^{1}=\frac{1}{i \hbar}\left\langle\left[P_{k}, V\right]\right\rangle_{\mathrm{q}}, \\
L_{k}^{21}=\frac{1}{\hbar^{2}} \int_{-\infty}^{0} d t_{1} e^{\varepsilon t_{1}}\left\langle\left[V\left(t_{1}\right),\left[P_{k}, V\right]\right]\right\rangle_{\mathrm{q}}, \\
L_{k}^{22}=\frac{1}{\hbar^{2}} \int_{-\infty}^{0} d t_{1} e^{\varepsilon t_{1}}\left\langle\left[V\left(t_{1}\right), i \hbar \sum_{l} P_{l} \frac{\partial L_{k}^{1}\left(\ldots,\left\langle P_{l}\right\rangle, \ldots\right)}{\partial\left\langle P_{l}\right\rangle}\right]\right\rangle_{\mathrm{q}} .
\end{gathered}
$$

Аналогичным образом можно найти члены, отвечающие высшим порядкам $V^{3}, V^{4}$ и т. д.

\section{1. Обобщенные кинетические уравнения для системы в термостате.} В работе [47] было дано обобщение уравнений (4.5) на случай системы, взаимодействующей с термостатом. Концепция термостата, или теплового резервуара, довольно непроста и имеет определенную специфику [48]. Стандартно термостат определяется как система, которая эффективно имеет бесконечное число степеней свободы. Термостатом является тепловой резервуар, поддерживающий заданную температуру изучаемой системы. Следуя Боголюбову [3], мы будем считать термостат источником стохастизации малой подсистемы (которая в предельном случае может быть одной частицей). Примерами таких систем может быть атомная или молекулярная система, взаимодействующая с электромагнитным полем, или система ядерных или электронных спинов, взаимодействующая с решеткой кристалла.

Будем описывать полную систему гамильтонианом

$$
H=H_{1}+H_{2}+V
$$

где

$$
H_{1}=\sum_{\alpha} E_{\alpha} a_{\alpha}^{\dagger} a_{\alpha}, \quad V=\sum_{\alpha, \beta} \Phi_{\alpha \beta} a_{\alpha}^{\dagger} a_{\beta}, \quad \Phi_{\alpha \beta}=\Phi_{\beta \alpha}^{\dagger} .
$$

Здесь $H_{1}$ - гамильтониан малой подсистемы и $a_{\alpha}^{\dagger}, a_{\alpha}$ - операторы рождения и уничтожения квазичастиц в малой подсистеме с энергиями $E_{\alpha} ; V$ - оператор взаимодействия между малой подсистемой и термостатом; $H_{2}$ - гамильтониан термостата, который мы не выписываем явно. Величины $\Phi_{\alpha \beta}$ являются операторами, действующими на переменные термостата. Мы предполагаем, что состояние системы можно охарактеризовать набором операторов $\left\langle P_{\alpha \beta}\right\rangle=\left\langle a_{\alpha}^{\dagger} a_{\beta}\right\rangle$, а состояние термостата - оператором $\left\langle H_{2}\right\rangle$. Здесь $\langle\cdot\rangle$ обозначает усреднение с НСО, который будет определен ниже.

Запишем квазиравновесный оператор в виде

$$
\rho_{\mathrm{q}}(t)=\exp (-S(t, 0))
$$


где

$$
S(t, 0)=\Omega(t)+\sum_{\alpha \beta} P_{\alpha \beta} F_{\alpha \beta}(t)+\beta H_{2}, \quad \Omega(t)=\ln \operatorname{Tr} \exp \left(-\sum_{\alpha \beta} P_{\alpha \beta} F_{\alpha \beta}(t)-\beta H_{2}\right) .
$$

Здесь $F_{\alpha \beta}(t)$ - термодинамические параметры, сопряженные с $P_{\alpha \beta}$, а $\beta$ - обратная температура термостата. Все операторы рассматриваются в представлении Гейзенберга. НСО запишем в виде

$$
\rho(t)=\exp (-\overline{S(t, 0)})
$$

где

$$
\overline{S(t, 0)}=\varepsilon \int_{-\infty}^{0} d t_{1} e^{\varepsilon t_{1}}\left(\Omega\left(t+t_{1}\right)+\sum_{\alpha \beta} P_{\alpha \beta} F_{\alpha \beta}(t)+\beta H_{2}\right) .
$$

Параметры $F_{\alpha \beta}(t)$ определяются из условия $\left\langle P_{\alpha \beta}\right\rangle=\left\langle P_{\alpha \beta}\right\rangle_{\mathrm{q}}$. При выводе кинетических уравнений мы используем разложение по параметру малого взаимодействия $V$; также предполагается, что справедливо равенство $\left\langle\Phi_{\alpha \beta}\right\rangle_{\mathrm{q}}=0$.

Для дальнейшего анализа удобно переписать $\rho_{\mathrm{q}}$ в виде

$$
\rho_{\mathrm{q}}=\rho_{1} \otimes \rho_{2}=Q_{\mathrm{q}}^{-1} \exp \left(-L_{0}(t)\right),
$$

где

$$
\begin{gathered}
\rho_{1}=Q_{1}^{-1} \exp \left(-\sum_{\alpha \beta} P_{\alpha \beta} F_{\alpha \beta}(t)\right), \quad Q_{1}=\operatorname{Tr} \exp \left(-\sum_{\alpha \beta} P_{\alpha \beta} F_{\alpha \beta}(t)\right), \\
\rho_{2}=Q_{2}^{-1} e^{-\beta H_{2},} \\
Q_{2}=\operatorname{Tr} \exp \left(-\beta H_{2}\right), \\
Q_{\mathrm{q}}=Q_{1} Q_{2}, \quad L_{0}=\sum_{\alpha \beta} P_{\alpha \beta} F_{\alpha \beta}(t)+\beta H_{2} .
\end{gathered}
$$

Исходным является соотношение

$$
\frac{d\left\langle P_{\alpha \beta}\right\rangle}{d t}=\frac{1}{i \hbar}\left\langle\left[P_{\alpha \beta}, H\right]\right\rangle=\frac{1}{i \hbar}\left(E_{\beta}-E_{\alpha}\right)\left\langle P_{\alpha \beta}\right\rangle+\frac{1}{i \hbar}\left\langle\left[P_{\alpha \beta}, V\right]\right\rangle,
$$

в котором мы ограничились членами второго порядка по $V$. Кинетические уравнения для системы в термостате для $\left\langle P_{\alpha \beta}\right\rangle$ имеют вид

$$
\frac{d\left\langle P_{\alpha \beta}\right\rangle}{d t}=\frac{1}{i \hbar}\left(E_{\beta}-E_{\alpha}\right)\left\langle P_{\alpha \beta}\right\rangle-\frac{1}{\hbar^{2}} \int_{-\infty}^{0} d t_{1} e^{\varepsilon t_{1}}\left\langle\left[\left[P_{\alpha \beta}, V\right], V\left(t_{1}\right)\right]\right\rangle_{\mathrm{q}} .
$$

Эти уравнения обобщают результаты работы [46] для системы в термостате. Можно показать, что предположение о модельной форме гамильтониана (4.8) является несущественным. Для произвольных $H_{1}$ и $V$ и некоторого набора переменных $\left\langle P_{k}\right\rangle$, которые удовлетворяют условию $\left[H_{1}, P_{k}\right]=\sum_{l} c_{k l} P_{l}$, можно построить квазиравновесный статистический оператор $\rho_{\mathrm{q}}$ в виде

$$
\rho_{\mathrm{q}}=Q_{\mathrm{q}}^{-1} \exp \left(-\sum_{k} P_{k} F_{k}(t)-\beta H_{2}\right)
$$


где $F_{k}(t)$ - параметры, сопряженные с $\left\langle P_{k}\right\rangle$. При этом кинетические уравнения для $\left\langle P_{k}\right\rangle$ имеют вид

$$
\frac{d\left\langle P_{k}\right\rangle}{d t}=\frac{i}{\hbar} \sum_{l} c_{k l}\left\langle P_{l}\right\rangle-\frac{1}{\hbar^{2}} \int_{-\infty}^{0} d t_{1} e^{\varepsilon t_{1}}\left\langle\left[\left[P_{k}, V\right], V\left(t_{1}\right)\right]\right\rangle_{\mathrm{q}} .
$$

Следует отметить, что полученные обобщенные кинетические уравнения служат полезным инструментом исследования квантовой динамики в конденсированных средах [15], [16], [18], [49]. Одним из методов исследования квантовой динамики является метод редуцированной (приведенной) матрицы плотности. В этом подходе приведенная матрица плотности получается посредством усреднения $\rho^{1}=\operatorname{Tr}_{2} \rho$ полной матрицы по нерелевантным степеням свободы термостата. Известным примером такого подхода являются уравнения Редфилда для спиновой матрицы плотности, которые также были получены (в обобщенной форме) с помощью метода $\mathrm{HCO}[15],[16]$, [49]. Уравнения типа Редфилда для $\left\langle P_{\alpha \beta}\right\rangle$ в общем виде записываются как

$$
\begin{aligned}
\frac{d\left\langle P_{\alpha \beta}\right\rangle}{d t}= & \frac{1}{i \hbar}\left(E_{\beta}-E_{\alpha}\right)\left\langle P_{\alpha \beta}\right\rangle- \\
& -\sum_{\nu}\left(K_{\beta \nu}\left\langle P_{\alpha \nu}\right\rangle+K_{\alpha \nu}^{\dagger}\left\langle P_{\nu \beta}\right\rangle\right)+\sum_{\mu, \nu} K_{\alpha \beta, \mu \nu}\left\langle P_{\mu \nu}\right\rangle .
\end{aligned}
$$

По своей структуре они соответствуют уравнениям Редфилда для спиновой матрицы плотности

$$
\frac{\partial \rho^{\alpha \alpha^{\prime}}}{\partial t}=-i \omega_{\alpha \alpha^{\prime}} \rho^{\alpha \alpha^{\prime}}+\sum_{\beta, \beta^{\prime}} R_{\alpha \alpha^{\prime} \beta \beta^{\prime}} \rho^{\beta \beta^{\prime}}
$$

в случае отсутствия внешнего поля (случай с переменным внешним полем рассмотрен в [15], [16], [49]). Здесь $\rho^{\alpha \alpha^{\prime}}$ есть матричный $\alpha \alpha^{\prime}$-элемент спиновой матрицы плотности, $\omega_{\alpha \alpha^{\prime}}=\left(E_{\alpha}-E_{\alpha^{\prime}}\right) / \hbar$, где $E_{\alpha}-$ энергия спинового состояния $\alpha$, a $R_{\alpha \alpha^{\prime} \beta \beta^{\prime}} \rho^{\beta \beta^{\prime}}$ есть так называемая “матрица релаксации”. Подробности обозначений см. в работах [15], [16].

Возвращаясь к уравнению (4.19), рассмотрим случай диагональных средних значений $\left\langle P_{\alpha \alpha}\right\rangle$. В результате получим

$$
\frac{d\left\langle P_{\alpha \alpha}\right\rangle}{d t}=\sum_{\nu} K_{\alpha \alpha, \nu \nu}\left\langle P_{\nu \nu}\right\rangle-\left(K_{\alpha \alpha}+K_{\alpha \alpha}^{\dagger}\right)\left\langle P_{\alpha \alpha}\right\rangle,
$$

где

$$
\begin{gathered}
K_{\alpha \alpha, \beta \beta}=\frac{1}{\hbar^{2}} J_{\alpha \beta, \beta \alpha}\left(\frac{E_{\alpha}-E_{\beta}}{\hbar}\right)=W_{\beta \rightarrow \alpha}, \\
K_{\alpha \alpha}+K_{\alpha \alpha}^{\dagger}=\frac{1}{\hbar^{2}} \sum_{\beta} J_{\beta \alpha, \alpha \beta}\left(\frac{E_{\beta}-E_{\alpha}}{\hbar}\right)=W_{\alpha \rightarrow \beta} .
\end{gathered}
$$

Величины $W_{\beta \rightarrow \alpha}$ и $W_{\alpha \rightarrow \beta}$ обозначают вероятности переходов, выраженные в терминах спектральных интенсивностей $J$. Используя свойства спектральных интенсивностей [5], можно убедиться в том, что вероятности переходов удовлетворяют уравнениям детального баланса

$$
\frac{W_{\beta \rightarrow \alpha}}{W_{\alpha \rightarrow \beta}}=\frac{e^{-\beta E_{\alpha}}}{e^{-\beta E_{\beta}}}
$$


В результате получаем уравнение

$$
\frac{d\left\langle P_{\alpha \alpha}\right\rangle}{d t}=\sum_{\nu} W_{\nu \rightarrow \alpha}\left\langle P_{\nu \nu}\right\rangle-\sum_{\nu} W_{\alpha \rightarrow \nu}\left\langle P_{\alpha \alpha}\right\rangle
$$

которое имеет обычный вид известного уравнения Паули (Pauli master equation) для матрицы плотности [15], [16].

\section{2. Уравнение типа Шредингера для динамической системы в термо-} стате. Рассмотрим теперь, следуя работам [15], [16], [50], поведение малой динамической подсистемы с гамильтонианом $H_{1}$, которая взаимодействует с термостатом с гамильтонианом $H_{2}$. В качестве операторов, характеризующих состояние малой подсистемы, выберем операторы $a_{\alpha}^{\dagger}, a_{\alpha}$, и $n_{\alpha}=a_{\alpha}^{\dagger} a_{\alpha}$. В этом случае квазиравновесный статистический оператор $\rho_{\mathrm{q}}$ принимает форму

$$
\rho_{\mathrm{q}}=\exp \left(\Omega-\sum_{\alpha}\left(f_{\alpha}(t) a_{\alpha}+f_{\alpha}^{\dagger}(t) a_{\alpha}^{\dagger}+F_{\alpha}(t) n_{\alpha}\right)-\beta H_{2}\right) \equiv \exp (-S(t, 0)),
$$

где

$$
\Omega=\ln \operatorname{Tr} \exp \left(-\sum_{\alpha}\left(f_{\alpha}(t) a_{\alpha}+f_{\alpha}^{\dagger}(t) a_{\alpha}^{\dagger}+F_{\alpha}(t) n_{\alpha}\right)-\beta H_{2}\right) .
$$

Здесь $f_{\alpha}, f_{\alpha}^{\dagger}$ и $F_{\alpha}$ играют роль множителей Лагранжа. Они являются параметрами, сопряженными с $\left\langle a_{\alpha}\right\rangle_{\mathrm{q}},\left\langle a_{\alpha}^{\dagger}\right\rangle_{\mathrm{q}}$ и $\left\langle n_{\alpha}\right\rangle_{\mathrm{q}}$ :

$$
\left\langle a_{\alpha}\right\rangle_{\mathrm{q}}=-\frac{\delta \Omega}{\delta f_{\alpha}(t)}, \quad\left\langle n_{\alpha}\right\rangle_{\mathrm{q}}=-\frac{\delta \Omega}{\delta F_{\alpha}(t)}, \quad \frac{\delta S}{\delta\left\langle a_{\alpha}\right\rangle_{\mathrm{q}}}=f_{\alpha}(t), \quad \frac{\delta S}{\delta\left\langle n_{\alpha}\right\rangle_{\mathrm{q}}}=F_{\alpha}(t) .
$$

Величины $a_{\alpha}, a_{\alpha}^{\dagger}$ в статистическом операторе можно интерпретировать как источники квантового шума [15], [16].

Квазиравновесный статистический оператор преобразуем к виду $\rho_{\mathrm{q}}=\rho_{1} \otimes \rho_{2}$, где

$$
\begin{gathered}
\rho_{1}=\exp \left(\Omega_{1}-\sum_{\alpha}\left(f_{\alpha}(t) a_{\alpha}+f_{\alpha}^{\dagger}(t) a_{\alpha}^{\dagger}+F_{\alpha}(t) n_{\alpha}\right)\right), \\
\Omega_{1}=\ln \operatorname{Tr} \exp \left(-\sum_{\alpha}\left(f_{\alpha}(t) a_{\alpha}+f_{\alpha}^{\dagger}(t) a_{\alpha}^{\dagger}+F_{\alpha}(t) n_{\alpha}\right)\right), \\
\rho_{2}=\exp \left(\Omega_{2}-\beta H_{2}\right), \quad \Omega_{2}=\ln \operatorname{Tr} \exp \left(-\beta H_{2}\right) .
\end{gathered}
$$

В результате для НСО $\rho$ получим выражение (4.11). Мы предполагаем, что выполнены условия

$$
\left\langle a_{\alpha}\right\rangle_{\mathrm{q}}=\left\langle a_{\alpha}\right\rangle, \quad\left\langle a_{\alpha}^{\dagger}\right\rangle_{\mathrm{q}}=\left\langle a_{\alpha}^{\dagger}\right\rangle, \quad\left\langle n_{\alpha}\right\rangle_{\mathrm{q}}=\left\langle n_{\alpha}\right\rangle
$$

Мы исходим из уравнений движения

$$
\begin{aligned}
& i \hbar \frac{d\left\langle a_{\alpha}\right\rangle}{d t}=\left\langle\left[a_{\alpha}, H_{1}\right]\right\rangle+\left\langle\left[a_{\alpha}, V\right]\right\rangle, \\
& i \hbar \frac{d\left\langle n_{\alpha}\right\rangle}{d t}=\left\langle\left[n_{\alpha}, H_{1}\right]\right\rangle+\left\langle\left[n_{\alpha}, V\right]\right\rangle .
\end{aligned}
$$


Во втором порядке по $V$ получаем

$$
\begin{aligned}
& i \hbar \frac{d\left\langle a_{\alpha}\right\rangle}{d t}=E_{\alpha}\left\langle a_{\alpha}\right\rangle+\frac{1}{i \hbar} \int_{-\infty}^{0} d t_{1} e^{\varepsilon t_{1}}\left\langle\left[\left[a_{\alpha}, V\right], V\left(t_{1}\right)\right]\right\rangle_{\mathrm{q}}, \\
& i \hbar \frac{d\left\langle n_{\alpha}\right\rangle}{d t}=\frac{1}{i \hbar} \int_{-\infty}^{0} d t_{1} e^{\varepsilon t_{1}}\left\langle\left[\left[n_{\alpha}, V\right], V\left(t_{1}\right)\right]\right\rangle_{\mathrm{q}} .
\end{aligned}
$$

Здесь $V\left(t_{1}\right)$ обозначает оператор $V$ в представлении взаимодействия. Разложение дает

$$
\begin{aligned}
& i \hbar \frac{d\left\langle a_{\alpha}\right\rangle}{d t}=E_{\alpha}\left\langle a_{\alpha}\right\rangle+ \\
& \quad+\frac{1}{i \hbar} \int_{-\infty}^{0} d t_{1} e^{\varepsilon t_{1}}\left(\sum_{\beta, \mu, \nu}\left\langle\Phi_{\alpha \beta} \phi_{\mu \nu}\left(t_{1}\right)\right\rangle_{\mathrm{q}}\left\langle a_{\beta} a_{\mu}^{\dagger} a_{\nu}\right\rangle_{\mathrm{q}}-\left\langle\phi_{\mu \nu}\left(t_{1}\right) \Phi_{\alpha \beta}\right\rangle_{\mathrm{q}}\left\langle a_{\mu}^{\dagger} a_{\nu} a_{\beta}\right\rangle_{\mathrm{q}}\right),
\end{aligned}
$$

где $\phi_{\mu \nu}\left(t_{1}\right)=\Phi_{\mu \nu}\left(t_{1}\right) \exp \left(\frac{i}{\hbar}\left(E_{\mu}-E_{\nu}\right) t_{1}\right)$, или, в другой форме,

$$
\begin{aligned}
i \hbar \frac{d\left\langle a_{\alpha}\right\rangle}{d t}=E_{\alpha}\left\langle a_{\alpha}\right\rangle & +\frac{1}{i \hbar} \sum_{\beta, \mu} \int_{-\infty}^{0} d t_{1} e^{\varepsilon t_{1}}\left\langle\Phi_{\alpha \mu} \phi_{\mu \beta}\left(t_{1}\right)\right\rangle_{\mathrm{q}}\left\langle a_{\beta}\right\rangle+ \\
& +\frac{1}{i \hbar} \sum_{\beta, \mu, \nu} \int_{-\infty}^{0} d t_{1} e^{\varepsilon t_{1}}\left\langle\left[\Phi_{\alpha \nu}, \phi_{\mu \nu}\left(t_{1}\right)\right]\right\rangle_{\mathrm{q}}\left\langle a_{\mu}^{\dagger} a_{\nu} a_{\beta}\right\rangle_{\mathrm{q}} .
\end{aligned}
$$

В результате получаем

$$
i \hbar \frac{d\left\langle a_{\alpha}\right\rangle}{d t}=E_{\alpha}\left\langle a_{\alpha}\right\rangle+\frac{1}{i \hbar} \sum_{\beta, \mu} \int_{-\infty}^{0} d t_{1} e^{\varepsilon t_{1}}\left\langle\Phi_{\alpha \mu} \phi_{\mu \beta}\left(t_{1}\right)\right\rangle_{\mathrm{q}}\left\langle a_{\beta}\right\rangle .
$$

С помощью спектральных представлений для корреляционных функций запишем уравнение

$$
i \hbar \frac{d\left\langle a_{\alpha}\right\rangle}{d t}=E_{\alpha}\left\langle a_{\alpha}\right\rangle+\sum_{\beta} K_{\alpha \beta}\left\langle a_{\beta}\right\rangle
$$

где $K_{\alpha \beta}$ определяется следующим образом:

$$
\frac{1}{i \hbar} \sum_{\mu} \int_{-\infty}^{0} d t_{1} e^{\varepsilon t_{1}}\left\langle\Phi_{\beta \mu} \phi_{\mu \nu}\left(t_{1}\right)\right\rangle_{\mathrm{q}}=\frac{1}{2 \pi} \sum_{\mu} \int_{-\infty}^{+\infty} d \omega \frac{J_{\mu \nu, \beta \mu}(\omega)}{\hbar \omega-E_{\mu}-E_{\nu}+i \varepsilon}=K_{\beta \nu} .
$$

Таким образом, мы получили уравнение типа Шредингера для средних амплитуд $\left\langle a_{\alpha}\right\rangle$. Оно является в определенном смысле аналогом (или обобщением) уравнения Шредингера для случая движения частицы в среде. Рассмотрим эту аналогию более подробно. Для этого запишем аналог волновой функции в виде

$$
\psi(\mathbf{r})=\sum_{\alpha} \chi_{\alpha}(\mathbf{r})\left\langle a_{\alpha}\right\rangle
$$

Здесь $\left\{\chi_{\alpha}(\mathbf{r})\right\}$ - полный ортонормированный набор одночастичных волновых функций оператора $\left(-\hbar^{2} / 2 m\right) \nabla^{2}+v(\mathbf{r})$ и $v(\mathbf{r})$ - потенциальная энергия. Мы имеем уравнение

$$
\left(-\frac{\hbar^{2}}{2 m} \nabla^{2}+v(\mathbf{r})\right) \chi_{\alpha}(\mathbf{r})=E_{\alpha} \chi_{\alpha}(\mathbf{r})
$$


Таким образом, величина $\psi(\mathbf{r})$ играет роль волновой функции для движения частицы в среде с трением. При этом уравнение (4.34) преобразуется к виду

$$
i \hbar \frac{\partial \psi(\mathbf{r})}{\partial t}=\left(-\frac{\hbar^{2}}{2 m} \nabla^{2}+v(\mathbf{r})\right) \psi(\mathbf{r})+\int d^{3} r^{\prime} K\left(\mathbf{r}, \mathbf{r}^{\prime}\right) \psi\left(\mathbf{r}^{\prime}\right)
$$

где ядро $K\left(\mathbf{r}, \mathbf{r}^{\prime}\right)$ интегрального уравнения (4.38) имеет вид

$$
K\left(\mathbf{r}, \mathbf{r}^{\prime}\right)=\sum_{\alpha, \beta} K_{\alpha \beta} \chi_{\alpha}(\mathbf{r}) \chi_{\beta}^{\dagger}\left(\mathbf{r}^{\prime}\right)=\frac{1}{i \hbar} \sum_{\alpha, \beta, \mu} \int_{-\infty}^{0} d t_{1} e^{\varepsilon t_{1}}\left\langle\Phi_{\alpha \mu} \phi_{\mu \beta}\left(t_{1}\right)\right\rangle_{\mathrm{q}} \chi_{\alpha}(\mathbf{r}) \chi_{\beta}^{\dagger}\left(\mathbf{r}^{\prime}\right)
$$

Видно, что уравнение (4.38) действительно можно назвать уравнением типа Шредингера для динамической системы в термостате. Интересно отметить, что очень похожие уравнения типа Шредингера с нелокальным взаимодействием применялись в теории столкновений [15], [16] для описания рассеяния частицы на совокупности многих рассеивающих центров. Для того чтобы показать яснее некоторые особенности уравнения (4.38), рассмотрим оператор трансляции $e^{i \mathbf{q p} / \hbar}$, где $\mathbf{q}=\mathbf{r}^{\prime}-\mathbf{r}$, $\mathbf{p}=-i \hbar \nabla_{r}$. Тогда уравнение (4.38) запишется в виде

$$
i \hbar \frac{\partial \psi(\mathbf{r})}{\partial t}=\left(-\frac{\hbar^{2}}{2 m} \nabla^{2}+v(\mathbf{r})\right) \psi(\mathbf{r})+\sum_{p} D(\mathbf{r}, \mathbf{p}) \psi(\mathbf{r})
$$

где

$$
D(\mathbf{r}, \mathbf{p})=\int d^{3} q K(\mathbf{r}, \mathbf{r}+\mathbf{q}) e^{i \mathbf{q p} / \hbar}
$$

Разумно предположить, что волновая функция $\psi(\mathbf{r})$ меняется не очень значительно на характерной корреляционной длине ядра $K\left(\mathbf{r}, \mathbf{r}^{\prime}\right)$. Тогда, используя разложение в ряд для $e^{i \mathbf{q p} / \hbar}$, получим в нулевом порядке

$$
i \hbar \frac{\partial \psi(\mathbf{r})}{\partial t}=\left(-\frac{\hbar^{2}}{2 m} \nabla^{2}+v(\mathbf{r})+\operatorname{Re} U(\mathbf{r})\right) \psi(\mathbf{r})+i \operatorname{Im} U(\mathbf{r}) \psi(\mathbf{r}),
$$

где

$$
U(\mathbf{r})=\operatorname{Re} U(\mathbf{r})+i \operatorname{Im} U(\mathbf{r})=\int d^{3} q K(\mathbf{r}, \mathbf{r}+\mathbf{q}) .
$$

Уравнение (4.41) имеет в точности функциональную форму уравнения Шредингера с комплексным потенциалом, известным в теории столкновений [15], [16]. Далее, разлагая $e^{i \mathbf{q p} / \hbar}$ в ряд вплоть до второго порядка, представим уравнение (4.38) в виде [16], [50]

$$
\begin{aligned}
i \hbar \frac{\partial \psi(\mathbf{r})}{\partial t}=\left\{\left(-\frac{\hbar^{2}}{2 m} \nabla^{2}\right.\right. & +v(\mathbf{r}))+U(\mathbf{r})-\frac{1}{i \hbar} \int d^{3} r^{\prime} K\left(\mathbf{r}, \mathbf{r}+\mathbf{r}^{\prime}\right) \mathbf{r}^{\prime} \mathbf{p}+ \\
& \left.+\frac{1}{2} \int d^{3} r^{\prime} K\left(\mathbf{r}, \mathbf{r}+\mathbf{r}^{\prime}\right) \sum_{i, k=1}^{3} r_{i}^{\prime} r_{k}^{\prime} \nabla_{i} \nabla_{k}\right\} \psi(\mathbf{r}) .
\end{aligned}
$$


Если ввести функцию

$$
\mathbf{A}(\mathbf{r})=\frac{c}{i \hbar e} \int d^{3} r^{\prime} K\left(\mathbf{r}, \mathbf{r}+\mathbf{r}^{\prime}\right) \mathbf{r}^{\prime}
$$

аналогичную в некотором смысле комплексному векторному потенциалу А электромагнитного поля, и тензор обратных эффективных масс [15], [51]

$$
\left\{\frac{1}{M(\mathbf{r})}\right\}_{i k}=\frac{1}{m} \delta_{i k}-\int d^{3} r^{\prime} \operatorname{Re} K\left(\mathbf{r}, \mathbf{r}+\mathbf{r}^{\prime}\right) r_{i}^{\prime} r_{k}^{\prime}
$$

то можно записать уравнение (4.43) в следующей форме:

$$
i \hbar \frac{\partial \psi(\mathbf{r})}{\partial t}=\left\{-\frac{\hbar^{2}}{2} \sum_{i, k}\left(\frac{1}{M(\mathbf{r})}\right)_{i k} \nabla_{i} \nabla_{k}+v(\mathbf{r})+U(\mathbf{r})+\frac{i \hbar e}{m c} \mathbf{A}(\mathbf{r}) \nabla+i T(\mathbf{r})\right\} \psi(\mathbf{r}),
$$

где

$$
T(\mathbf{r})=\frac{1}{2} \int d^{3} r^{\prime} \operatorname{Im} K\left(\mathbf{r}, \mathbf{r}+\mathbf{r}^{\prime}\right) \sum_{i, k} r_{i}^{\prime} r_{k}^{\prime} \nabla_{i} \nabla_{k}
$$

В изотропной среде тензор $\{1 / M(\mathbf{r})\}_{i k}$ диагонален и $\mathbf{A}(\mathbf{r})=0$. Необходимо отметить, что понятие тензора эффективных масс было введено в физику в работе Блохинцева и Нордгейма [52] (см. также обзор [51]).

Полезно пояснить сказанное на языке физики твердого тела. Для этого рассмотрим собственные функции $\psi_{n}(\mathbf{k}, \mathbf{r})$ электронов в поле периодической решетки, которые имеют вид (здесь $n$ - номер полосы)

$$
\psi_{n}\left(\mathbf{k}, \mathbf{r}+\mathbf{R}_{l}\right)=e^{i \mathbf{k R}_{l}} \psi_{n}(\mathbf{k}, \mathbf{r})(\mathbf{k}, \mathbf{r}), \quad \psi_{n}(\mathbf{k}, \mathbf{r})=e^{i \mathbf{k r}} u_{n}(\mathbf{k}, \mathbf{r})
$$

Они называются блоховскими функциями, а описываемые ими электроны в кристалле соответственно называются блоховскими электронами. Форма блоховских функций дает указание на физический смысл вектора k: электрон в кристалле может быть представлен в виде плоской волны, модулированной с периодом решетки. Исследование общих свойств функции $E_{n}(k)$ вблизи выделенных точек зоны $\mathbf{k}_{0}$ приводит к соотношению [15], [51]

$$
E_{n}(\mathbf{k})=E_{n}\left(\mathbf{k}_{0}\right)+\frac{\hbar}{m} \mathbf{s p}_{n n}+\frac{\hbar^{2} \mathbf{s}^{2}}{2 m}+\frac{\hbar^{2}}{m^{2}} \sum_{j \neq n} \frac{\left(\mathbf{s p}_{n j}\right)\left(\mathbf{s p}_{j n}\right)}{E_{n}\left(\mathbf{k}_{0}\right)-E_{j}\left(\mathbf{k}_{0}\right)}+\cdots
$$

где величины $\mathbf{p}_{n j}$ и $\mathbf{s}$ определены в работах [15], [51]. Данное соотношение показывает, что электрон, описываемый уравнением Шредингера с периодическим потенциалом, нужно рассматривать как частицу, подверженную влиянию взаимодействия с потенциалом, или квазичастицу. Свойства этой квазичастицы уже содержат взаимодействие со статической решеткой.

Рассмотрим случай, когда точка $\mathbf{k}_{0}$ соответствует экстремуму. Для второй производной от энергии по $\mathbf{k}$ мы имеем

$$
\frac{m}{\hbar^{2}} \frac{\partial^{2} E_{n}}{\partial s_{\alpha} \partial s_{\beta}}=\frac{m}{\hbar^{2}} \frac{\partial^{2} E_{n}}{\partial k_{\alpha} \partial k_{\beta}}=\delta_{\alpha \beta}+\frac{1}{m} \sum_{j \neq n} \frac{p_{n j}^{\alpha} p_{j n}^{\beta}+p_{n j}^{\beta} p_{j n}^{\alpha}}{E_{n}\left(\mathbf{k}_{0}\right)-E_{j}\left(\mathbf{k}_{0}\right)}
$$


Здесь $s_{\alpha}$ и $s_{\beta}$ - декартовы координаты вектора $\mathbf{s}$ относительно некоторой фиксированной системы осей, а $p_{n j}^{\alpha}$ - соответствующие компоненты матричного элемента оператора импульса. С помощью соотношения

$$
\left(\frac{m}{m^{*}}\right)_{\alpha \beta}=\frac{m}{\hbar^{2}} \frac{\partial^{2} E_{n}}{\partial k_{\alpha} \partial k_{\beta}}
$$

вводится в рассмотрение тензор обратной эффективной массы. Тогда (4.50) запишется следующим образом:

$$
\left(\frac{m}{m^{*}}\right)_{\alpha \beta}=\delta_{\alpha \beta}+\frac{1}{m} \sum_{j \neq n} \frac{p_{n j}^{\alpha} p_{j n}^{\beta}+p_{n j}^{\beta} p_{j n}^{\alpha}}{E_{n}\left(\mathbf{k}_{0}\right)-E_{j}\left(\mathbf{k}_{0}\right)} .
$$

Его часто называют правилом сумм для эффективных масс; иногда оно называется также правилом $f$-сумм. Диагональные элементы в (4.52) имеют более простой вид:

$$
\left(\frac{m}{m^{*}}\right)_{\alpha \alpha}=1+\frac{2}{m} \sum_{j \neq n} \frac{\left|p_{n j}^{\alpha}\right|^{2}}{E_{n}\left(\mathbf{k}_{0}\right)-E_{j}\left(\mathbf{k}_{0}\right)} .
$$

Отсюда видно, что взаимодействие данного энергетического уровня с более низкими уровнями или с состояниями ионного остова, для которых $E_{j}<E_{n}$, приводит к уменьшению эффективной массы, в то время как взаимодействие с вышележащими состояниями $\left(E_{j}>E_{n}\right)$ увеличивает ее.

Понятие эффективной массы достаточно хорошо определено вблизи минимума или максимума зоны. При этом эффективная масса $m^{*}$ вблизи минимума

$$
E=E_{0}+\frac{k_{x}^{2} \hbar^{2}}{2 m^{*}}
$$

отличается от эффективной массы $m^{* *}$ вблизи потолка зоны

$$
E=E_{1}+\frac{\left(k_{x}-\pi / a\right)^{2} \hbar^{2}}{2 m^{* *}} .
$$

В обоих случаях массы $m^{*}$ и $m^{* *}$ могут сильно отличаться от истинной массы электрона. Так, если энергетическая зона очень узка и кривизна энергетической поверхности у дна зоны мала, то эффективная масса $m^{*}$ велика, иногда значительно больше истинной. С другой стороны, у потолка зоны, если кривизна велика, масса $m^{* *}$ очень мала. Тензорный характер эффективной массы, вследствие которого электрон или дырка могут ускоряться различным образом в зависимости от направления силы, важен для кристаллов с несимметричной структурой или в случае, когда минимум или максимум лежит не в центре зоны Бриллюэна. В некоторых случаях нужно считать величины $m_{x}^{*}, m_{y}^{*}, m_{z}^{*}$ отрицательными; при этом вблизи седловой точки некоторые из них будут положительными, а некоторые - отрицательными. Интересно отметить близкое сходство этого механизма с теорией позитрона Дирака. В настоящее время изучен целый ряд систем, например двумерный графитовый слой, в которых движение электронов описывается в терминах релятивистской квантовой физики; при этом электрон ведет себя, как релятивистская частица с нулевой массой. 
Концепция эффективной массы получила очень широкое распространение, в особенности в физике полупроводников и полупроводниковых устройств [15], [51], теории полярона [53], полупроводниковых сверхрешеток [15], [51], микроэлектронике и физике наноструктур [15], [51]. Выяснилось, что понятие эффективной массы оказывается исключительно полезным в теории проводимости, а также в других областях физики твердого тела, в ядерной физике и т. д.

В теории систем многих взаимодействующих частиц рассматривается общая концепция квазичастиц [15], которые представляют собой спектр элементарных возбуждений системы при учете электрон-электронного, электрон-фононного и других взаимодействий. Квазичастица представляет собой ренормированную (одетую облакам виртуальных частиц) "затравочную” (или голую) частицу с эффективной массой вида $m^{*}=m\left(1+\lambda_{e-e}+\lambda_{e-\mathrm{ph}}\right)$. При этом перенормированная (эффективная) масса может отличаться от исходной массы очень значительно, как, например, для систем с тяжелыми фермионами [15].

В некоторых задачах теории столкновений [15] концепция эффективной массы позволяет записать обобщенное уравнение Шредингера, в котором вместо реальной массы $m$ стоит эффективная масса $m^{*}$, зависящая от положения частицы (такая же ситуация реализуется в некоторых полупроводниковых гетероструктурах). В ядерной физике использование нелокального оптического потенциала $V\left(\mathbf{r}, \mathbf{r}^{\prime}\right)$ приводит при определенных условиях к волновому уравнению для локального потенциала $V(\mathbf{r})$ с эффективной массой вида [15]

$$
M^{*}(r)=M\left(1-\frac{M \alpha^{2}}{\hbar^{2}} V(r)\right)^{-1} .
$$

В заключение заметим, что линейное уравнение типа Шредингера является достаточно хорошим приближением для системы бозе-частиц, если выполнено условие

$$
\left\langle n_{\alpha}\right\rangle-\left|\left\langle a_{\alpha}\right\rangle\right|^{2}=\left(e^{F_{\alpha}}-1\right)^{-1} \ll 1
$$

что по существу соответствует условию $\left\langle n_{\alpha}\right\rangle \ll 1$. Это отвечает учету только слабо возбужденных состояний в системе квазичастиц. В случае статистики Ферми нельзя исключить линейные члены сдвигом операторов на комплексное число, так как это преобразование не является каноническим. В квантовой теории поля источники, линейные по ферми-операторам, вводятся с помощью классических антикоммутирующих как друг с другом, так и с первичными полями, спинорных полей. Этот более сложный случай рассматривался нами в работе [54].

Отметим также, что введение величины $\psi(\mathbf{r})$ не означает, что состояние малой динамической подсистемы становится чистым состоянием. Оно остается смешанным, так как описывается статистическим оператором; при этом динамика системы описывается системой связанных уравнений эволюции для величин $f_{\alpha}, f_{\alpha}^{\dagger}$ и $F_{\alpha}$. Попытки вывода уравнения типа Шредингера для частицы в среде предпринимались неоднократно. Однако ясно видно, что уравнение (4.38) типа уравнения Шредингера с затуханием наиболее адекватно описывает динамическое поведение частицы в среде с учетом диссипации. Это обуславливает многочисленные применения данного уравнения к задачам физики, физической химии, биофизики и т. п. Подробное обсуждение различных аспектов диссипативного поведения и стохастических процессов в сложных системах дано в работах [15], [16]. 


\section{5. ЭФФЕКТЫ ЗАТУХАНИЯ В ОТКРЫТОЙ ДИНАМИЧЕСКОЙ СИСТЕМЕ}

В работе [55] применимость развитого общего метода к конкретным задачам была продемонстрирована на примере вычисления естественной ширины спектральных линий атомной системы [15]. Хорошо известно, что возбужденные уровни изолированной атомной системы, которые проявляются в результате взаимодействия с собственным электромагнитным полем, обладают конечным временем жизни. Это приводит к тому, что уровни становятся квазидискретными, приобретая конечную малую ширину, которая и называется естественной шириной спектральных линий. Следовательно, ширина спектральной линии равна обратной величине времени жизни возбужденного состояния.

Будем рассматривать атом, взаимодействующий только с собственным электромагнитным полем, в приближении, когда атом можно считать покоящимся. Для простоты предположим, что атом может находиться всего в двух состояниях, основном и возбужденном, которые мы обозначим индексами $\alpha=1,2$, причем энергии $E_{\alpha}$ - это относительные энергии этих состояний. Таким образом, мы полагаем, что атомная система в возбужденном состоянии играет роль малой "неравновесной" подсистемы, а электромагнитное поле можно рассматривать как аналог термостата. Релаксация, которая в данном случае является распадом возбужденного уровня, происходит путем переходов с излучением. Гамильтониан полной системы запишем в виде $H=H_{\mathrm{at}}+H_{\mathrm{f}}+V$. Здесь

$$
H_{\mathrm{at}}=\sum_{\alpha} E_{\alpha} a_{\alpha}^{\dagger} a_{\alpha}
$$

есть гамильтониан атомной подсистемы, $a_{\alpha}^{\dagger}$ и $a_{\alpha}$ - операторы рождения и уничтожения для системы в состоянии $E_{\alpha}$ и

$$
H_{\mathrm{f}}=\sum_{k, \lambda} k c b_{k, \lambda}^{\dagger} b_{k, \lambda}
$$

есть гамильтониан поперечного электромагнитного поля [15], [55], где $\hbar \mathbf{k}-$ импульс фотона, $c$ - скорость света, $\lambda$ - индекс поляризации, $b_{k, \lambda}^{\dagger}$ и $b_{k, \lambda}$ - операторы рождения и уничтожения фотона в состоянии $(\mathbf{k} \lambda), \lambda=1,2$. Оператор взаимодействия $V$, отвечающий за переходы с излучением, в нерелятивистском приближении имеет вид

$$
V=-\frac{e}{m c} \mathbf{p} \mathbf{A}_{\mathrm{tr}}(\mathbf{r})
$$

где $e$ и $m$ - заряд и масса электрона, $\mathbf{A}_{\mathrm{tr}}(\mathbf{r})$ - вектор-потенциал поперечного электромагнитного поля излучения, взятый в точке $\mathbf{r}$, причем $\left[\mathbf{p} \times \mathbf{A}_{\mathrm{tr}}(\mathbf{r})\right]=0$. Векторпотенциал $\mathbf{A}_{\mathrm{tr}}(\mathbf{r})$ можно разложить в ряд по плоским волнам:

$$
\mathbf{A}_{\mathrm{tr}}(\mathbf{r})=\frac{1}{\sqrt{\Omega}} \sum_{k, \lambda}\left(\frac{2 \pi \hbar^{2} c}{k}\right)^{1 / 2} \mathbf{e}_{k, \lambda}\left(b_{k, \lambda} e^{i \mathbf{k r} / \hbar}+b_{k, \lambda}^{\dagger} e^{-i \mathbf{k r} / \hbar}\right) .
$$

Суммирование по $\mathbf{k}$ производится по всем состояниям плоских волн с импульсом $\hbar \mathbf{k}$ в конечном ящике объемом $\Omega$. Суммирование по $\lambda$ производится по двум дозволенным направлениям поляризации фотона с импульсом $\hbar \mathbf{k}$. Единственные неисчезающие матричные элементы операторов рождения и уничтожения $b_{k, \lambda}^{\dagger}$ и $b_{k, \lambda}$ можно 
представить в виде

$$
\left\langle n_{k, \lambda}-1\left|b_{k, \lambda}\right| n_{k, \lambda}\right\rangle=\sqrt{n_{k, \lambda}}, \quad\left\langle n_{k, \lambda}+1\left|b_{k, \lambda}^{\dagger}\right| n_{k, \lambda}\right\rangle=\sqrt{n_{k, \lambda}} .
$$

Запишем теперь оператор взаимодействия $V$ в виде (4.9):

$$
V=\sum_{\alpha, \beta} \varphi_{\alpha \beta} a_{\alpha}^{\dagger} a_{\beta}, \quad \varphi_{\alpha \beta}=\varphi_{\beta \alpha}^{\dagger}
$$

где

$$
\begin{gathered}
\varphi_{\alpha \beta}=\frac{1}{\sqrt{\Omega}} \sum_{k, \lambda}\left(G_{\alpha, \beta}(k, \lambda) b_{k, \lambda}+b_{k, \lambda}^{\dagger} G_{\beta \alpha}^{*}(k, \lambda)\right), \\
G_{\alpha, \beta}(k, \lambda)=-\frac{e}{m c}\left(\frac{2 \pi \hbar^{2} c}{k}\right)^{1 / 2} \mathbf{e}_{k, \lambda}\left\langle\alpha\left|e^{i \mathbf{k r} / \hbar} \mathbf{p}\right| \beta\right\rangle .
\end{gathered}
$$

Здесь $|\alpha\rangle$ и $|\beta\rangle$ - собственные векторы гамильтониана $H_{\text {at }}$ с собственными значениями $E_{\alpha}$ и $E_{\beta}$,

$$
H_{\text {at }}|\alpha\rangle=E_{\alpha}|\alpha\rangle
$$

Будем, как обычно, предполагать, что разрешен электрический дипольный переход для распада из состояния $a=1$ в состояние $b=2$, т. е. будем считать, что $\mathbf{k r} \ll 1$. Тогда $\varphi_{\alpha \beta}$ примет вид

$$
\varphi_{\alpha \beta}=-\frac{e}{m c}\langle\alpha|\mathbf{p}| \beta\rangle \sum_{k, \lambda}\left(\frac{2 \pi \hbar^{2} c}{k}\right)^{1 / 2} \mathbf{e}_{k, \lambda}\left(b_{k, \lambda}+b_{k, \lambda}^{\dagger}\right) .
$$

Матричные элементы оператора дипольного момента $\mathbf{d}=e \mathbf{r}$ между состояниями $|\alpha\rangle$ и $|\beta\rangle$ связаны с матричными элементами импульса р следующим образом:

$$
\langle\alpha|\mathbf{p}| \beta\rangle=-\frac{m}{e \hbar}\left(E_{\alpha}-E_{\beta}\right) \mathbf{d}_{\alpha \beta} .
$$

В настоящей работе мы считаем, что атом не обладает стационарным дипольным моментом, т. е. $\langle\alpha|\mathbf{p}| \alpha\rangle=0$.

Воспользуемся теперь полученным выше уравнением типа Шредингера с затуханием

$$
i \hbar \frac{d\left\langle a_{\alpha}\right\rangle}{d t}=E_{\alpha}\left\langle a_{\alpha}\right\rangle+\sum_{\beta} K_{\alpha \beta}\left\langle a_{\beta}\right\rangle
$$

где

$$
K_{\alpha \beta}=\frac{1}{i \hbar} \sum_{\gamma} \int_{-\infty}^{0} d t_{1} e^{\varepsilon t_{1}}\left\langle\varphi_{\alpha \gamma} \tilde{\varphi}_{\gamma \beta}\left(t_{1}\right)\right\rangle_{\mathrm{q}} .
$$

Здесь $\tilde{\varphi}_{\alpha \beta}(t)$ имеет вид

$$
\tilde{\varphi}_{\alpha \beta}(t)=\varphi_{\alpha \beta}(t) \exp \left(\frac{i}{\hbar}\left(E_{\alpha}-E_{\beta}\right) t\right) .
$$

Из сказанного выше ясно, что $K_{a a}=0$ и $K_{b a}=0$. Поэтому имеем

$$
i \hbar \frac{d\left\langle a_{b}\right\rangle}{d t}=E_{b}\left\langle a_{b}\right\rangle+K_{b b}\left\langle a_{b}\right\rangle,
$$


где

$$
K_{b b}=\frac{2 \pi \hbar^{2} e^{2}}{m^{2} c} \frac{1}{\Omega} \sum_{k} \int_{-\infty}^{\infty} d \omega \frac{1}{k} \frac{J(k, \omega)}{\hbar \omega_{0}+\hbar \omega+i \varepsilon} A_{a b}^{a b}\left(\frac{\mathbf{k}}{k}\right), \quad \hbar \omega_{0}=E_{b}-E_{a} .
$$

Здесь введены обозначения

$$
\begin{gathered}
J(k, \omega)=\left(\left\langle n_{k}\right\rangle+1\right) \delta(\omega+c k)+\left\langle n_{k}\right\rangle \delta(\omega-c k), \\
\left\langle n_{k}\right\rangle=\sum_{\lambda}\left\langle n_{k \lambda}\right\rangle=\left(e^{\beta c k}-1\right)^{-1}=n(k),
\end{gathered}
$$

и

$$
A_{a b}^{a b}=|\langle a|\mathbf{p}| b\rangle|^{2}-\left(\langle a|\mathbf{p}| b\rangle \frac{\mathbf{k}}{k}\right)\left(\langle b|\mathbf{p}| a\rangle \frac{\mathbf{k}}{k}\right)
$$

Далее имеем

$$
\begin{aligned}
& \frac{1}{\Omega} \sum_{k} \int_{-\infty}^{\infty} d \omega \frac{1}{k} \frac{J(k, \omega)}{\hbar \omega_{0}+\hbar \omega+i \varepsilon} A_{a b}^{a b}\left(\frac{\mathbf{k}}{k}\right)= \\
& \quad=\frac{1}{(2 \pi)^{3}} \int k d k \int_{-\infty}^{\infty} d \omega \frac{1}{k} \frac{J(k, \omega)}{\hbar \omega_{0}+\hbar \omega+i \varepsilon} \int d \varpi A_{a b}^{a b}\left(\frac{\mathbf{k}}{k}\right)
\end{aligned}
$$

где $d \varpi$ - элемент телесного угла. Можно проверить равенство

$$
\int d \varpi A_{a b}^{a b}\left(\frac{\mathbf{k}}{k}\right)=\frac{8 \pi}{3}|\langle a|\mathbf{p}| b\rangle|^{2}
$$

Для величины $K_{b b}$ найдем, что (здесь $\nu=c k$ )

$$
K_{b b}=\frac{2 e^{2}}{m^{2} c^{2} \hbar}|\langle a|\mathbf{p}| b\rangle|^{2} \int_{0}^{\infty} \nu d \nu\left(\frac{n(\nu)+1}{\omega_{0}-\nu+i \varepsilon}+\frac{n(\nu)}{\omega_{0}+\nu+i \varepsilon}\right),
$$

откуда при $T=0 \mathrm{~K}$ найдем выражение для затухания $\Gamma_{b}$ :

$$
K_{b b}=\Delta E_{b}-\frac{i \hbar}{2} \Gamma_{b}, \quad \Gamma_{b}=\frac{4}{3} \frac{e^{2} \omega_{0}}{m^{2} c^{3} \hbar}|\langle a|\mathbf{p}| b\rangle|^{2}=\frac{4}{3} \frac{\omega_{0}^{3}}{c^{3} \hbar}\left|\mathbf{d}_{a b}\right|^{2} .
$$

Это выражение совпадает с известным значением естественной ширины спектральных линий атома [15]. Таким образом, с помощью уравнения типа Шредингера с затуханием можно просто вычислять сдвиг энергии и затухание в различных конкретных системах.

Полезно отметить, что задача о естественной ширине спектральных линий многократно использовалась для тестирования различных новых расчетных схем и теорий квантовой физики [15]. Так, в работе [56] отмечалось: “Разнообразие трактовок проблемы уширения линий настолько велико, что сбивает с толку, и часто трудно увидеть, описывают или не описывают различные подходы одни и те же эффекты". Задачу о естественной ширине спектральных линий рассматривал также Блохинцев [57] в 1946 г. Его работа носит название "Вычисление естественной ширины спектральных линий стационарным методом". Автор пишет: “Обычно задачу об испускании и поглощении света рассматривают методом квантовых переходов. Между 
тем эта задача, подобно задаче о дисперсии, может быть крайне просто решена методом стационарных состояний". Далее автор записывает систему уравнений для амплитуд состояний двух типов: когда излучатель находится в состоянии $m$ и световые кванты отсутствуют и когда излучатель находится в состоянии $n$ и излучен один световой квант. С учетом закона сохранения энергии получается решение для амплитуды и на его основе приближенное выражение для положения уровня всей системы (излучатель и излучение). Из этого выражения следует “... в точности то смещение и размытые уровни, которые получает Дирак при расчете резонансного рассеяния". Далее находится "спектральное распределение в пределах ширины линии". Блохинцев замечает, что при преобразовании амплитуды к координатному представлению "получим расходящуюся волну, амплитуда которой медленно нарастает по мере удаления от источника излучения так же, как это имеет место для классического затухающего осциллятора".

В нашем подходе использовалась концепция "квантового шума", позволившая построить НСО с "источниками" шума и за счет этого получить систему связанных уравнений для средних амплитуд и плотностей. По существу полученная система уравнений аналогична в определенном смысле системе уравнений Блохинцева для амплитуд и моделирует поведение "затухающего осциллятора" [57]. Роль трения играет взаимодействие $\varphi_{\alpha \beta}$ малой системы со средой (термостатом).

\section{6. ОБОБЩЕННАЯ ФОРМУЛА ВАН ХОВА}

В этом разделе мы рассмотрим кратко, следуя работе [58], проблему рассеяния медленных нейтронов в неравновесной среде. Известно, что микроскопическое описание динамического поведения конденсированных сред (газов, жидкостей и твердых тел) использует понятие пространственно-временных корреляций и соответствующих им корреляционных функций [5], [15], [59]. Метод рассеяния медленных нейтронов [15], [59] является эффективным инструментом изучения статических (структура) и динамических (спектры квазичастичных возбуждений) свойств сложных многочастичных систем. Основополагающее значение для описания рассеяния нейтронов в конденсированных средах, находящихся в состоянии статистического равновесия, имела теория Ван Хова [15], [59]. Ван Хов в работах [15], [59] вывел известную формулу, позволившую выразить сечение рассеяния медленных нейтронов в терминах фурье-образов пространственно-временных корреляционных функций. В работе [58] было дано обобщение теории Ван Хова для случая рассеяния медленных нейтронов на системах, находящихся в неравновесном состоянии (например, при наличии градиентов вещества, температуры и т. п.). При этом использовался подход, описанный в предыдущих разделах.

Напомним, что основной величиной, которая измеряется в экспериментах по рассеянию частиц [15], [59], является дифференциальное сечение рассеяния. В качестве примера сначала рассмотрим кристалл с постоянной решетки, равной $a$. Амплитуда перехода для частицы, падающей на мишень, есть величина первого порядка по величине взаимодействия между частицей и мишенью. Вероятность перехода из начального состояния в конечное под влиянием потенциала $V$ записывается в виде

$$
W_{k k^{\prime}}=\frac{2 \pi}{\hbar}\left|\int d^{3} r \psi_{k^{\prime}}^{*} V \psi_{k}\right|^{2} D_{k^{\prime}}\left(E^{\prime}\right),
$$


где $D_{k^{\prime}}\left(E^{\prime}\right)$ есть плотность конечных состояний рассеянной частицы. Сечение рассеяния определим как

$$
d \sigma=\frac{W_{k k^{\prime}}}{\text { величина падающего потока }} .
$$

Падающий поток равен $\hbar k^{\prime} / m$, а плотность конечных состояний рассеянной частицы

$$
D_{k^{\prime}}\left(E^{\prime}\right)=\frac{1}{(2 \pi)^{3}} \frac{d^{3} k^{\prime}}{d E^{\prime}}=\frac{m^{2}}{(2 \pi)^{3} \hbar^{3}} d \Omega\left(\frac{\hbar k^{\prime}}{m}\right) .
$$

Дифференциальное сечение рассеяния при этом переписывается в виде

$$
\frac{d \sigma}{d \Omega}=\frac{m^{2}}{(2 \pi)^{2} \hbar^{4}} \frac{k^{\prime}}{k}\left|\int d^{3} r e^{i\left(\mathbf{k}^{\prime}-\mathbf{k}\right) \mathbf{r} / \hbar} V(\mathbf{r})\right|^{2} .
$$

Применим теперь данный подход для описания неупругого рассеяния медленных нейтронов [15], [58]. Эксперимент заключается в рассеянии на образце монохроматического потока нейтронов с энергией $E$ и волновым вектором $\mathbf{k}$. Рассеянные нейтроны характеризуются их конечной энергией $E^{\prime}=E+\hbar \omega$ и направлением $\Omega$ их конечного волнового вектора $\mathbf{k}^{\prime}$. Нас интересует величина $I$, которая равна числу нейтронов с волновыми векторами, лежащим между $\mathbf{k}$ и $\mathbf{k}+d \mathbf{k}$, рассеянных в единицу времени:

$$
I=I_{0} \frac{m a^{3}}{\hbar k} d w\left(\mathbf{k} \rightarrow \mathbf{k}^{\prime}\right) D(\mathbf{k}) d \mathbf{k} .
$$

Здесь $m$ - масса нейтрона, $a^{3}$ - объем элементарной ячейки мишени и $d w\left(\mathbf{k} \rightarrow \mathbf{k}^{\prime}\right)-$ вероятность перехода из начального состояния $|\mathbf{k}\rangle$ в конечное состояние $\left|\mathbf{k}^{\prime}\right\rangle$, а $D(\mathbf{k})$ плотность состояний, которая равна

$$
D(\mathbf{k}) d \mathbf{k}=\frac{a^{3}}{(2 \pi)^{3}} k^{2} d \boldsymbol{\Omega} d k .
$$

Для волновых функций падающих и рассеянных частиц имеем

$$
\psi_{k}=\sqrt{\frac{m}{k}} e^{i \mathbf{k r} / \hbar}, \quad \psi_{k^{\prime}}=\frac{1}{(2 \pi \hbar)^{3 / 2}} e^{i \mathbf{k}^{\prime} \mathbf{r} / \hbar} .
$$

Амплитуда перехода для падающей на мишень частицы равна

$$
d w\left(\mathbf{k} \rightarrow \mathbf{k}^{\prime}\right)=\frac{m}{\hbar^{2} k} \frac{d^{3} k}{(2 \pi \hbar)^{3}} \int_{-\infty}^{\infty} d t d^{3} r d^{3} r^{\prime}\left\langle V(\mathbf{r}) V\left(\mathbf{r}^{\prime}, t\right)\right\rangle e^{-i\left(\mathbf{k}-\mathbf{k}^{\prime}\right)\left(\mathbf{r}-\mathbf{r}^{\prime}\right) / \hbar-i \omega t} .
$$

Другими словами, амплитуда перехода, которая описывает изменение в единицу времени состояния падающего пучка частиц, может быть записана как

$$
d w\left(\mathbf{k} \rightarrow \mathbf{k}^{\prime}\right)=\frac{1}{\hbar^{2}} \int_{-\infty}^{\infty} d t \operatorname{Tr}\left(\rho^{\mathrm{m}} V_{\mathbf{k}^{\prime} \mathbf{k}}(0) V_{\mathbf{k}^{\prime} \mathbf{k}}(t)\right) e^{-i \omega t},
$$

где $\rho^{\mathrm{m}}$ есть статистический оператор, описывающий образец (мишень).

Сечение рассеяния имеет вид

$$
\frac{d^{2} \sigma}{d \Omega d E^{\prime}}=\frac{1}{d \Omega d \omega} \frac{I}{I_{0}}
$$


или, в несколько иной форме,

$$
\frac{d^{2} \sigma}{d \Omega d E^{\prime}}=A \int_{-\infty}^{\infty} d t d^{3} r d^{3} r^{\prime}\left\langle V(\mathbf{r}) V\left(\mathbf{r}^{\prime}, t\right)\right\rangle e^{-\frac{i}{\hbar}\left(\mathbf{k}-\mathbf{k}^{\prime}\right)\left(\mathbf{r}-\mathbf{r}^{\prime}\right)-i \omega t},
$$

где

$$
A=\frac{m^{2}}{(2 \pi)^{3} \hbar^{5}} \frac{k^{\prime}}{k}, \quad E^{\prime}=\frac{\left(k^{\prime}\right)^{2}}{2 m} .
$$

Обычно для оператора взаимодействия нейтрона со средой принимается выражение [15], [59]

$$
V=\frac{2 \pi \hbar^{2}}{m} \sum_{i} b_{i} \delta\left(\mathbf{r}-\mathbf{R}_{i}\right)
$$

Здесь $\mathbf{R}_{i}$ - оператор положения ядра атома мишени и $b_{i}-$ соответствующая длина рассеяния. Необходимо также принять во внимание, что

$$
V=\sum_{i=1}^{N} V\left(\mathbf{r}-\mathbf{R}_{i}\right)=\sum_{i=1}^{N} e^{-i \mathbf{p} \mathbf{R}_{i} / \hbar} V(\mathbf{r}) e^{i \mathbf{p} \mathbf{R}_{i} / \hbar}
$$

и

$$
\left\langle\beta \mathbf{k}^{\prime}|V| \alpha \mathbf{k}\right\rangle=\left\langle\mathbf{k}^{\prime}|V(\mathbf{r})| \mathbf{k}\right\rangle \sum_{i=1}^{N}\left\langle\beta\left|e^{-i \mathbf{k}^{\prime} \mathbf{R}_{i} / \hbar} e^{i \mathbf{k R}_{i} / \hbar}\right| \alpha\right\rangle .
$$

Отсюда получаем

$$
\frac{d^{2} \sigma}{d \Omega d E^{\prime}} \propto \frac{k^{\prime}}{k} \frac{1}{2 \pi} \sum_{i, j} \int_{-\infty}^{\infty} d t \frac{1}{N} b_{i} b_{j}\left\langle e^{i \boldsymbol{\kappa} \mathbf{R}_{i}(0) / \hbar} e^{-i \boldsymbol{\kappa} \mathbf{R}_{j}(t) / \hbar}\right\rangle e^{-i \omega t}
$$

где $\kappa=k-k^{\prime}$. Данное выражение описывает суть теории Ван Хова [15], [59], позволяющей записать сечение неупругого рассеяния медленных нейтронов в терминах пространственно-временных корреляций. Как указывалось выше, в данном случае усреднение проводится с равновесной матрицей плотности (статистическим оператором) $\rho^{\mathrm{m}}$, т. е. $\langle\bullet\rangle=\operatorname{Tr}\left(\rho^{\mathrm{m}} \bullet\right)$.

Рассмотрим теперь случай неравновесной среды и воспользуемся методом НСО. Гамильтониан среды (мишени) будем обозначать как $H_{\mathrm{m}}$, гамильтониан падающего пучка нейтронов - как $H_{\mathrm{b}}$ и оператор взаимодействия - как $V$. Полный гамильтониан имеет вид

$$
H=H_{0}+V=H_{\mathrm{m}}+H_{\mathrm{b}}+V .
$$

Амплитуда перехода записывается как

$$
d w\left(\mathbf{k} \rightarrow \mathbf{k}^{\prime}\right)=\frac{1}{\hbar^{2}} \int_{-\infty}^{\infty} d t \operatorname{Tr}_{m}\left(\rho^{\mathrm{m}}(t) V_{\mathbf{k}^{\prime} \mathbf{k}}(0) V_{\mathbf{k}^{\prime} \mathbf{k}}(t)\right) e^{-i \omega t}
$$

где $\rho^{\mathrm{m}}(t)$ - НСО среды (мишени). В соответствии со сказанным выше сечение рассеяния равно

$$
\frac{d^{2} \sigma}{d \Omega d E^{\prime}}=A \int_{-\infty}^{\infty} d t d^{3} r d^{3} r^{\prime}\left\langle V(\mathbf{r}) V\left(\mathbf{r}^{\prime}, t\right)\right\rangle_{\mathrm{m}} e^{-i\left(\mathbf{k}-\mathbf{k}^{\prime}\right)\left(\mathbf{r}-\mathbf{r}^{\prime}\right) / \hbar-i \omega t},
$$

где $\langle\cdot\rangle_{\mathrm{m}}=\operatorname{Tr}_{m}\left(\rho^{\mathrm{m}}(t) \bullet\right)$ и $A, E^{\prime}$ определены в $(6.12)$. 
Примем во внимание, что

$$
\left\langle\alpha^{\prime} \mathbf{k}^{\prime}|V| \alpha \mathbf{k}\right\rangle=\left\langle\mathbf{k}^{\prime}|V(\mathbf{r})| \mathbf{k}\right\rangle \sum_{i=1}^{N}\left\langle\alpha^{\prime}\left|e^{-i\left(\mathbf{k}^{\prime} \mathbf{R}_{i}\right) / \hbar} e^{i\left(\mathbf{k} \mathbf{R}_{i}\right) / \hbar}\right| \alpha\right\rangle .
$$

В результате получим [15], [58]

$$
\begin{aligned}
& \frac{d^{2} \sigma}{d \Omega d E^{\prime}}=-\frac{1}{(i \hbar)^{2}} \tilde{A} \sum_{i, j=1}^{N} \int_{0}^{t} d \tau \times \\
& \times \sum_{\alpha}\left\langle\alpha\left|\left\{e^{i \boldsymbol{\kappa} \mathbf{R}_{i}(\tau-t) / \hbar} e^{i \boldsymbol{\kappa} \mathbf{R}_{j}(0) / \hbar} e^{i \omega(\tau-t)}+e^{i \boldsymbol{\kappa} \mathbf{R}_{i}(0) / \hbar} e^{i \boldsymbol{\kappa} \mathbf{R}_{j}(\tau-t) / \hbar} e^{-i \omega(\tau-t)}\right\} \rho^{\mathrm{m}}(t)\right| \alpha\right\rangle
\end{aligned}
$$

или, в другой форме,

$$
\frac{d^{2} \sigma}{d \Omega d E^{\prime}}=-\frac{1}{(i \hbar)^{2}} \tilde{A} \sum_{i, j=1}^{N} \int_{0}^{t} d \tau 2 \operatorname{Re}\left\langle e^{\frac{i}{\hbar} \kappa \mathbf{R}_{i}(\tau-t)} e^{\frac{i}{\hbar} \boldsymbol{\kappa} \mathbf{R}_{j}(0)}\right\rangle_{\mathrm{m}} e^{i \omega(\tau-t)} .
$$

Это выражение можно записать в терминах оператора плотности $n_{\boldsymbol{\kappa}}=\sum_{i}^{N} e^{i \kappa \mathbf{R}_{i} / \hbar}$ :

$$
\frac{d^{2} \sigma}{d \Omega d E^{\prime}}=\tilde{A} \cdot 2 \operatorname{Re} \mathcal{S}(\kappa, \omega, t)
$$

где

$$
\mathcal{S}(\boldsymbol{\kappa}, \omega, t)=-\frac{1}{(i \hbar)^{2}} \int_{0}^{t} d \tau e^{i \omega(\tau-t)}\left\langle n_{\boldsymbol{\kappa}}(\tau-t) n_{-\boldsymbol{\kappa}}\right\rangle_{\mathrm{m}}
$$

называется функиией рассеяния.

НCO среды (мишени) $\rho^{\mathrm{m}}(t)$ запишем как

$$
\begin{aligned}
\rho^{\mathrm{m}} & =\overline{\rho_{\mathrm{q}}(t, 0)}=\varepsilon \int_{-\infty}^{0} d \tau e^{\varepsilon \tau} \rho_{\mathrm{q}}(t+\tau, \tau)= \\
& =\varepsilon \int_{-\infty}^{0} d \tau e^{\varepsilon \tau} \exp \left(-\frac{H_{\mathrm{m}} \tau}{i \hbar}\right) \rho_{\mathrm{q}}(t+\tau, 0) \exp \left(\frac{H_{\mathrm{m}} \tau}{i \hbar}\right)= \\
& =\varepsilon \int_{-\infty}^{0} d \tau e^{\varepsilon \tau} \exp (-S(t+\tau, \tau)) .
\end{aligned}
$$

Другая форма этого выражения такова:

$$
\begin{aligned}
\rho^{\mathrm{m}}(t, 0)= & \exp (-S(t, 0))+ \\
& +\int_{-\infty}^{0} d \tau e^{\varepsilon \tau} \int_{0}^{t} d \tau^{\prime} \exp \left(-\tau^{\prime} S(t+\tau, \tau)\right) \dot{S}(t+\tau, \tau) \exp \left(-\left(\tau^{\prime}-t\right) S(t+\tau, \tau)\right),
\end{aligned}
$$

где

$$
\dot{S}(t, \tau)=\exp \left(-\frac{H_{\mathrm{m}} \tau}{i \hbar}\right) \dot{S}(t, 0) \exp \left(\frac{H_{\mathrm{m}} \tau}{i \hbar}\right)
$$

И

$$
\dot{S}(t, 0)=\frac{\partial S(t, 0)}{\partial t}+\frac{1}{i \hbar}[S(t, 0), H]=\sum_{m}\left(\dot{P}_{m} F_{m}(t)+\left(P_{m}-\left\langle\dot{P}_{m}\right\rangle_{q}^{t}\right) \dot{F}_{m}(t)\right)
$$


Окончательно выражение для функции рассеяния пучка нейтронов на неравновесной среде имеет вид [15], [58]

$$
\begin{aligned}
\mathcal{S}(\boldsymbol{\kappa}, \omega, t)= & -\frac{1}{(i \hbar)^{2}} \int_{0}^{t} d \tau\left\langle n_{\kappa}(\tau-t) n_{-\kappa}(0)\right\rangle_{\mathrm{q}}^{t} e^{i \omega(\tau-t)}- \\
& -\frac{1}{(i \hbar)^{2}} \int_{0}^{t} d \tau \int_{-\infty}^{0} d \tau^{\prime} e^{\varepsilon \tau^{\prime}}\left(n_{\kappa}(\tau-t) n_{-\kappa}(0), \dot{S}\left(t+\tau^{\prime}\right)\right)^{t+\tau^{\prime}} e^{i \omega(\tau-1)} .
\end{aligned}
$$

Здесь

$$
\rho_{\mathrm{q}}(t, 0)=\exp (-S(t, 0)), \quad\langle B\rangle_{q}^{t}=\operatorname{Tr}\left(B \rho_{\mathrm{q}}(t, 0)\right)
$$

и используется стандартное обозначение [5]

$$
(A, B)^{t}=\int_{0}^{1} d \tau \operatorname{Tr}\left[A \exp (-\tau S(t, 0))\left(B-\langle B\rangle_{q}^{t}\right) \exp ((\tau-1) S(t, 0))\right] .
$$

Следует подчеркнуть, что основной особенностью полученного выражения (6.27) для $\mathcal{S}(\boldsymbol{\kappa}, \omega, t)$ является наличие в нем члена $\dot{S}\left(t+\tau^{\prime}\right)$, описывающего изменение энтропии. В случае статистического равновесия изменение энтропии равно нулю, и в формуле (6.27) остается только первое слагаемое, описывающее рассеяние пучка нейтронов в равновесной среде.

Отметим, что задача о рассеянии нейтронов в неравновесной среде и нахождении $\mathrm{HCO}$ среды имеет ряд общих черт с рассмотренной выше задачей об описании малой подсистемы, взаимодействующей с термостатом. Действительно, состояние полной системы в момент времени $t$ описывается статистическим оператором вида

$$
\rho(t)=\exp \left(\frac{-i H_{0} t}{\hbar}\right) \rho(0) \exp \left(\frac{i H_{0} t}{\hbar}\right), \quad \rho(0)=\rho^{\mathrm{m}}(0) \otimes \rho^{\mathrm{b}}(0) .
$$

Начальное состояние среды (мишени) и падающего пучка частиц предполагает факторизованную форму оператора $\rho(0)$ в виде произведения статистического оператора среды (мишени) $\rho^{\mathrm{m}}(0)$ и статистического оператора пучка $\rho^{\mathrm{b}}(0)$. В момент времени $t$ эти операторы записываются как

$$
\begin{aligned}
& \rho^{\mathrm{b}}(t)=\operatorname{Tr}_{\mathrm{m}}[\rho(t)]=\operatorname{Tr}_{\mathrm{m}}\left(\exp \left(-\frac{i H_{0} t}{\hbar}\right) \rho^{\mathrm{m}}(0) \otimes \rho^{\mathrm{b}}(0) \exp \left(\frac{i H_{0} t}{\hbar}\right)\right), \\
& \rho^{\mathrm{m}}(t)=\operatorname{Tr}_{\mathrm{b}}[\rho(t)]=\operatorname{Tr}_{\mathrm{b}}\left(\exp \left(-\frac{i H_{0} t}{\hbar}\right) \rho^{\mathrm{m}}(0) \otimes \rho^{\mathrm{b}}(0) \exp \left(\frac{i H_{0} t}{\hbar}\right)\right)
\end{aligned}
$$

где $\operatorname{Tr}_{\mathrm{m}}$ и $\operatorname{Tr}_{\mathrm{b}}$ обозначает частичное усреднение по переменным среды и пучка соответственно.

Общее выражение для вероятности перехода из начального состояния статистической системы, описываемого статистическим оператором $\rho^{\mathrm{i}}$, в состояние с $\rho^{\mathrm{f}}$ дается формулой

$$
W_{\text {if }}(t)=\operatorname{Tr}\left(\rho^{\mathrm{i}}(t) \rho^{\mathrm{f}}(t)\right) .
$$

Разумно предположить, что $\rho^{\mathrm{i}}$ можно записать в форме $\rho^{\mathrm{i}}(t)=\rho^{\mathrm{i}}(0)=|k\rangle\langle k|$. В этом случае вероятность перехода в единицу времени примет вид

$$
w_{\text {if }}(t)=\frac{d}{d t} \operatorname{Tr}\left(|k\rangle\langle k| \rho^{f}(t)\right)=\frac{d}{d t}\left\langle k\left|\rho^{\mathrm{f}}(t)\right| k\right\rangle=\left\langle k\left|\frac{d}{d t} \rho^{\mathrm{f}}(t)\right| k\right\rangle .
$$


В свою очередь обобщенное уравнение Лиувилля с источниками в правой части для статистического оператора полной системы (среда + пучок) $\rho(t)$ запишется как

$$
\frac{\partial}{\partial t} \rho(t)-\frac{1}{i \hbar}\left[\left(H_{\mathrm{m}}+H_{\mathrm{b}}+V\right), \rho(t)\right]_{-}=-\varepsilon(\rho(t)-P \rho(t)) .
$$

Здесь $P$ - проекционный супероператор, обладающий следующими свойствами:

$$
P^{2}=P, \quad P(1-P)=0, \quad P(A+B)=P A+P B .
$$

Простейшим является случай, когда

$$
P \rho(t)=\rho^{\mathrm{m} 0} \otimes \rho^{\mathrm{b}}=\rho^{\mathrm{m} 0} \sum_{\alpha}\langle\alpha|\rho(t)| \alpha\rangle .
$$

Здесь $\rho^{\mathrm{m0}}-$ равновесный статистический оператор среды.

Применим теперь для описании неравновесной среды прием наложения граничных условий, описанный выше. Имеем

$$
\frac{\partial}{\partial t} \rho(t)-\frac{1}{i \hbar}\left[\left(H_{\mathrm{m}}+H_{\mathrm{b}}+V\right), \rho(t)\right]_{-}=-\varepsilon\left(\rho(t)-\rho^{\mathrm{m}}(t) \rho^{\mathrm{b}}(t)\right)
$$

где

$$
\rho^{\mathrm{m}}(t)=\operatorname{Tr}_{\mathrm{b}} \rho(t)=\sum_{k}\langle k|\rho(t)| k\rangle
$$

и (в общем случае)

$$
\rho^{\mathrm{b}}=\operatorname{Tr}_{\mathrm{m}}(\rho(t))=\sum_{\alpha}\langle\alpha|\rho(t)| \alpha\rangle=\sum_{k, k^{\prime}}\left\langle k\left|\rho^{\mathrm{b}}(t)\right| k\right\rangle|k\rangle\left\langle k^{\prime}\left|=\sum_{k, k^{\prime}} \rho_{k^{\prime} k}^{\mathrm{b}}\right| k\right\rangle\left\langle k^{\prime}\right| .
$$

Поэтому согласно методу НСО мы можем переписать уравнение (6.34) в виде

$$
\frac{\partial}{\partial t} \rho(t)-\frac{1}{i \hbar}[H, \rho(t)]_{-}=-\varepsilon\left(\rho(t)-\rho^{\mathrm{m}}(t) \sum_{q} \rho_{q q}^{\mathrm{b}}(t)|q\rangle\langle q|\right),
$$

где мы ограничились для $\rho^{\mathrm{b}}$ диагональными членами по состояниям $|q\rangle$, а $\varepsilon \rightarrow 0$ после термодинамического предельного перехода. В этом случае НСО примет вид

$$
\begin{aligned}
\rho_{\varepsilon}=\rho_{\varepsilon}(t, 0)=\overline{\rho_{\mathrm{q}}(t, 0)} & =\varepsilon \int_{-\infty}^{0} d \tau e^{\varepsilon \tau} \rho_{\mathrm{q}}(t+\tau, \tau)= \\
& =\varepsilon \int_{-\infty}^{0} d \tau U(\tau) \rho^{\mathrm{m}}(t) \sum_{k} \rho_{k k}^{\mathrm{b}}(t+\tau)|k\rangle\langle k| U^{\dagger}(\tau) .
\end{aligned}
$$

Здесь $U(t)$ - оператор эволюции.

По аналогии с выводом уравнений эволюции для малой подсистемы, взаимодействующей со средой [17], [47], [50], мы рассматриваем пучок нейтронов как малую подсистему, взаимодействующую со статистической средой в неравновесном состоянии. Мы можем записать соответствующее уравнение эволюции в виде

$$
\begin{aligned}
\frac{\partial}{\partial t} \rho_{q q}^{\mathrm{b}}(t)= & -\frac{1}{i \hbar} \varepsilon \int_{-\infty}^{0} d \tau e^{\varepsilon \tau} \times \\
& \times \sum_{k} \rho_{k k}^{\mathrm{b}}(t+\tau) \sum_{\alpha}\left\langle\alpha\left|\left\langle q\left|\left[U(\tau) \rho^{\mathrm{m}}(t)|k\rangle\langle k| U^{\dagger}(\tau), V\right]_{-}\right| q\right\rangle\right| \alpha\right\rangle .
\end{aligned}
$$


Следуя указанной аналогии, разумно ограничиться приближением низшего порядка и считать, что можно не учитывать эффекты памяти: $\rho_{k k}^{\mathrm{b}}(t+\tau) \simeq \rho_{k k}^{\mathrm{b}}(t)$. После интегрирования по частям наше уравнение эволюции примет вид

$$
\begin{aligned}
\frac{\partial}{\partial t} \rho_{q q}^{\mathrm{b}}(t)= & \frac{1}{\hbar^{2}} \sum_{k} \rho_{k k}^{\mathrm{b}}(t) \times \\
& \times \int_{-\infty}^{0} d \tau e^{\varepsilon \tau} \sum_{\alpha}\left\langle\alpha\left|\left\langle q\left|\left[U(\tau)\left[V(\tau), \rho^{\mathrm{m}}(t)|k\rangle\langle k|\right]_{-} U^{\dagger}(\tau), V\right]_{-}\right| q\right\rangle\right| \alpha\right\rangle .
\end{aligned}
$$

Как и при выводе кинетических уравнений для системы в термостате мы ограничиваемся вторым порядком по взаимодействию $V$. Из этого следует, что в уравнении (6.43) можно положить $U=U^{\dagger}=1$. В результате получаем уравнение, аналогичное уравнению Паули (4.24):

$$
\frac{\partial}{\partial t} \rho_{k k}^{\mathrm{b}}(t)=\sum_{q} W_{q \rightarrow k} \rho_{q q}^{\mathrm{b}}(t)-\sum_{q} W_{k \rightarrow q} \rho_{k k}^{\mathrm{b}}(t) .
$$

Здесь $W_{q \rightarrow k}$ являются эффективными вероятностями переходов, которые имеют вид

$$
W_{q \rightarrow k}=2 \operatorname{Re} \frac{1}{\hbar^{2}} \int_{-\infty}^{0} d \tau e^{\varepsilon \tau}\left\langle V_{q k} V_{k q}(\tau)\right\rangle_{\mathrm{m}}^{t},
$$

где $V_{q k}=\langle q|V| k\rangle,\langle\cdot\rangle_{\mathrm{m}}^{t}=\operatorname{Tr}\left(\bullet \rho^{\mathrm{m}}(t)\right)$ и $\varepsilon \rightarrow 0$ после термодинамического предельного перехода. Таким образом, мы получили обобщение выражений (6.1), (6.9) и (6.17) на случай неравновесной среды. Это дает прямое обоснование формулы (6.23).

\section{7. ЗАКЛЮЧЕНИЕ}

В представленной работе мы кратко проанализировали некоторые проблемы обоснования метода НСО [5] и показали его гибкость и эффективность при рассмотрении конкретных прикладных задач неравновесной статистической механики. В частности, мы обсудили вывод обобщенных кинетических уравнений. Основное внимание было уделено задаче о взаимодействии малой неравновесной подсистемы со средой (термостатом). Были проанализированы ее эволюция во времени и диссипативные процессы, имеющие место в результате взаимодействия со средой. С помощью метода НСО для динамической системы, слабо взаимодействующей с термостатом, было получено уравнение типа уравнения Шредингера с затуханием. При этом использовалась вспомогательная концепция "квантового шума", позволившая построить $\mathrm{HCO}$ с "источниками" шума и за счет этого получить систему связанных уравнений для средних амплитуд и плотностей.

В качестве иллюстрации была рассмотрена задача о естественной ширине спектральных линий. Данная проблема возникает при рассмотрении возбужденных уровней изолированной атомной системы, которые, появляясь с ненулевой вероятностью из-за взаимодействия с собственным электромагнитным полем, обладают конечным временем жизни. Это приводит к тому, что уровни становятся квазидискретными, приобретая конечную малую ширину, которая и называется естественной шириной спектральных линий. Было показано, что полученная система уравнений 
аналогична системе уравнений для амплитуд состояний и моделирует поведение "затухающего осциллятора". Роль трения играет взаимодействие малой системы со средой (термостатом). Получена также обобщенная формула Ван Хова для задачи рассеяния нейтронов в неравновесной среде. Более подробное и полное обсуждение этих и других вопросов неравновесной статистической механики содержится в работах [15], [16], [39]. Дальнейшее развитие метода НСО и его разнообразные приложения изложены в книгах [5], [14].

Благодарности. Автор выражает свою глубокую призательность Л. А. Покровскому и К. Валясеку за обсуждение и сотрудничество и отмечает их значительный вклад в теорию обобщенных кинетических уравнений.

\section{Список литературы}

[1] Н. Н. Боголюбов, Проблемы динамической теории в статистической физике, Гостехтеориздат, М., Л., 1946.

[2] N. N. Bogoliubov, "Problems of dynamical theory in statistical physics", Studies in Statistical Mechanics, v. I, eds. J. de Boer, G. E. Uhlenbeck, North-Holland, Amsterdam, 1962, $1-118$.

[3] Н. Н. Боголюбов, "О стохастических процессах в динамических системах", ЭЧАЯ, 9:4 (1978), 501-579.

[4] D. Ya. Petrina, Stochastic Dynamics and Boltzmann Hierarchy, De Gruyter Expositions in Mathematics, 48, Walter de Gruyter, Berlin, 2009.

[5] Д. Н. Зубарев, Неравновесная статистическая термодинамика, Наука, М., 1971.

[6] J. A. McLennan, Introduction to Nonequilibrium Statistical Mechanics, Prentice Hall, New York, 1989.

[7] B. C. Eu, Nonequilibrium Statistical Mechanics. Ensemble Method, Fundamental Theories of Physics, 93, Kluwer, Dordrecht, 1998.

[8] R. Zwanzig, "Time-correlation functions and transport coefficients in statistical mechanics", Ann. Rev. Phys. Chem., 16 (1965), 67-102.

[9] R. Zwanzig, "The concept of irreversibility in statistical mechanics", Pure and Appl. Chem., 22:3-4 (1970), 371-378.

[10] R. Zwanzig, Nonequilibrium Statistical Mechanics, Oxford Univ. Press, Oxford, New York, 2001.

[11] G. Gallavotti, Nonequilibrium and Irreversibility, Springer, Cham, 2014.

[12] В. В. Козлов, Ансамбли Гиббса и неравновеснал статистическал механика, РХД, М., 2008.

[13] V. Vedenyapin, A. Sinitsyn, E. Dulov, Kinetic Boltzmann, Vlasov and Related Equations, Elsevier, Amsterdam, 2011.

[14] Д. Н. Зубарев, В. Г. Морозов, Г. Рёпке, Статистическая механика неравновесных процессов, Физматлит, М., 2002.

[15] A. L. Kuzemsky, Statistical Mechanics and the Physics of Many-Particle Model Systems, World Sci., Singapore, 2017.

[16] A. L. Kuzemsky, "Theory of transport processes and the method of the nonequilibrium statistical operator", Internat. J. Modern Phys. B, 21:17 (2007), 2821-2949.

[17] A.L. Kuzemsky, "Generalized kinetic and evolution equations in the approach of the nonequilibrium statistical operator", Internat. J. Modern Phys. B, 19:6 (2005), 1029-1059.

[18] A. L. Kuzemsky, "Electronic transport in metallic systems and generalized kinetic equations", Internat. J. Modern Phys. B, 25:23n24 (2011), 3071-3183.

[19] M. Toda, R. Kubo, N. Saitô, Statistical Physics I. Equilibrium Statistical Mechanics, Springer, Berlin, 1992. 
[20] M. Toda, R. Kubo, N. Saitô, Statistical Physics II. Nonequilibrium Statistical Mechanics, Springer, Berlin, 1991.

[21] J. W. Gibbs, Elementary Principles in Statistical Mechanics: Developed with Especial Reference to the Rational Foundations of Thermodynamics, Dover, New York, 1960.

[22] Н.Н.Боголюбов, Д. Я. Петрина, Б. И. Хацет, "Математическое описание равновесного состояния классических систем на основе формализма канонического ансамбля", TMФ, 1:2 (1969), 251-274.

[23] A. L. Kuzemsky, "Thermodynamic limit in statistical physics", Internat. J. Modern Phys. B, 28:9 (2014), 1430004, 28 pp.

[24] Р. А. Минлос, Введение в математическую статистическую физику, МЦНМО, М., 2002.

[25] R. Zwanzig, "Ensemble method in the theory of irreversibility", J. Chem. Phys., 33:5 (1960), 1338-1341.

[26] P. G. Bergmann, J. L. Lebowitz, "New approach to nonequilibrium processes", Phys. Rev., 99:2 (1955), 578-587.

[27] J. L. Lebowitz, P. G. Bergmann, "Irreversible Gibbsian ensembles", Ann. Phys. (N. Y.), 1:1 (1957), 1-23.

[28] J. L. Lebowitz, "Stationary nonequilibrium Gibbsian ensembles", Phys. Rev., 114:5 (1959), $1192-1202$.

[29] J.L. Lebowitz, A. Shimony, "Statistical mechanics of open systems", Phys. Rev., 128:4 (1962), 1945-1958.

[30] Н. Н. Боголюбов, Ю.А. Митропольский, Асимптотические методы в теории нелинейных колебаний, Наука, М., 1974.

[31] Ю.А. Митропольский, Метод усреднения в нелинейной механике, Наукова думка, Киев, 1971.

[32] А. М. Самойленко, "Н.Н.Боголюбов и нелинейная механика", УМН, 49:5(299) (1994), 103-146.

[33] В.И. Арнольд, “От усреднения до статфизики”, Проблемы современной математической физики, Сборник статей. К 90-летию со дня рождения академика Николая Николаевича Боголюбова, Тр. МИАН, 228, Наука, М., 2000, 196-202.

[34] Н.Н.Боголюбов, "О некоторых проблемах, связанных с обоснованием статистической механики", История и методология естественных наук. Физика., 30, Изд-во Моск. ун-та, М., 1983, 3-8.

[35] Н. Н. Боголюбов, Д. Н. Зубарев, "Метод асимптотического приближения для систем с вращающейся фазой и его применение к движению заряженных частиц", Укр. матем. журн., 7:1 (1955), 5-17.

[36] В.В. Козлов, О.Г. Смолянов, "Информационная энтропия в задачах классической и квантовой статистической механики", Докл. РАН, 411:5 (2006), 587-590.

[37] E. T. Jaynes, Probability Theory: The Logic of Science, Cambridge Univ. Press, New York, 2003.

[38] L. M. Martyushev, V.D. Seleznev, "Maximum entropy production principle in physics, chemistry and biology", Phys. Rep., 426:1 (2006), 1-45.

[39] A. L. Kuzemsky, "Probability, information and statistical physics", Internat. J. Theor. Phys., 55:3 (2016), 1378-1404.

[40] Н.Н. Боголюбов, "Квазисредние в задачах статистической механики", Cтатистическая физика и квантовая теория поля, ред. Н. Н. Боголюбов, Наука, М., 1973, 7-80.

[41] Д. Н. Зубарев, "Граничные условия для статистических операторов в теории неравновесных процессов и квазисредние", ТМФ, 3:2 (1970), 276-286.

[42] A. L. Kuzemsky, "Bogoliubov's vision: quasiaverages and broken symmetry to quantum protectorate and emergence", Internat. J. Modern Phys. B, 24:8 (2010), 835-935. 
[43] Д. Н. Зубарев, В. П. Калашников, “Экстремальные свойства неравновесного статистического оператора", ТМФ, 1:1 (1969), 137-149.

[44] Д. Н. Зубарев, В.П. Калашников, "Построение статистических операторов для неравновесных процессов", ТМФ, 3:1 (1970), 126-134.

[45] D. N. Zubarev, V.P. Kalashnikov, "Derivation of the nonequilibrium statistical operator from the extremum of the information entropy", Physica, 46:4 (1970), 550-554.

[46] Л. А. Покровский, "Получение обобщенных кинетических уравнений с помощью неравновесного статистического оператора", Докл. АН СCCP, 183:4 (1968), 806-809.

[47] К. Валясек, А. Л. Куземский, "Кинетические уравнения для системы в термостате", TMФ, 4:2 (1970), 267-276.

[48] V.V. Kozlov, "Gibbs ensembles, equidistribution of the energy of sympathetic oscillators and statistical models of thermostat", Regul. Chaotic Dyn., 13:3 (2008), 141-154.

[49] A. L. Kuzemsky, "Statistical theory of spin relaxation and diffusion in solids", J. Low Temp. Phys., 143:5-6 (2006), 213-256.

[50] К. Валясек, Д. Н. Зубарев, А. Л. Куземский, "Уравнение типа Шредингера с затуханием для динамической системы в термостате", ТМФ, 5:2 (1970), 281-292.

[51] А.Л. Куземский, "Работы Д. И. Блохинцева и развитие квантовой физики”, ЭЧАЯ, 39:1 (2008), 5-81.

[52] Д. И. Блохинцев, Избранные труды, т. 1, Физматлит, М., 2009.

[53] Н. Н. Боголюбов, Н. Н. Боголюбов (мл.), Аспекты теории полярона, Физматлит, М., 2004.

[54] A. L. Kuzemsky, A. Pawlikowski, "Note on the diagonalization of a quadratic linear form defined on the set of second quantization fermion operators", Rep. Math. Phys., 3:3 (1972), 201-207.

[55] A. L. Kuzemsky, K. Walasek, "On the calculation of the natural width of spectral lines of atom by the methods of nonequilibrium statistical mechanics", Lett. Nuovo Cimento, 2 (1971), 953-956.

[56] S. Bloom, H. Margenau, "Quantum theory of spectral line broadening", Phys. Rev., 90:5 (1953), 791-794.

[57] Д. И. Блохинцев, “Вычисление естественной ширины спектральных линий стационарным методом”, ЖЖЭТФ, 16:11 (1946), 965-967.

[58] A. L. Kuzemsky, "Generalized Van Hove formula for scattering of neutrons by the nonequilibrium statistical medium", Internat. J. Modern Phys. B, 26:13 (2012), 1250092, 34 pp.

[59] W. Marshall, S. W. Lovesey, Theory of Thermal Neutron Scattering, Oxford Univ. Press, Oxford, 1971.

Поступила в редакцию 1.04.2017, после доработки 1.05.2017 